\title{
Coexisting Linear and Widely Linear Transceivers in the MIMO Interference Channel
}

\author{
Sandra Lagen, Student Member, IEEE, Adrian Agustin, Member, IEEE, and Josep Vidal, Member, IEEE
}

\begin{abstract}
Recent results have shown the benefits of widely linear precoding (WLP) in the MIMO interference channel (MIMO IC) assuming that all transmitters can follow the same strategy. Motivated by a transitional scenario where legacy linear transmitters coexist with widely linear ones, this work investigates the general $K$-user MIMO IC in a heterogeneous (linear and widely linear) transmitter deployment. In particular, we address the maximization of the weighted sum-rate (WSR) for (widely) linear transmit filters design through the use of the complexvalued formulation. Since the maximum WSR problem is nonconvex, and thus difficult to be solved, we formulate an equivalent minimum weighted mean square error problem that allows deriving closed-form expressions for (widely) linear transceivers. Then an iterative procedure is proposed, which is proven to reach a stationary point of the maximum WSR problem. Simulations show that the proposed procedure allows increasing the sumrate as compared to coordinated linear transceiver schemes. The gains are larger and significant in two different non-exclusive conditions: as the interference level increases or when the number of antennas is low.
\end{abstract}

Index Terms-MIMO interference channel, heterogeneous transceivers, improper Gaussian signaling, widely linear precoding, widely linear estimation, weighted sum-rate maximization.

\section{INTRODUCTION}

$\mathbf{T}$ HE $K$-user multiple-input multiple-output interference channel (MIMO IC) is a generic model for cellular communication systems that consists of $K$ transmitter-receiver pairs, each equipped with multiple antennas. All transmitters wish to send independent streams to its intended receiver simultaneously, such that interference is generated towards unintended receivers. Unfortunately, the optimal transmit/receive strategy with linear filters that maximizes the weighted sumrate (WSR) of the system is not known. From an optimization theory perspective, the problem is non-convex and NP-hard even in the single-antenna case [1]. Even so, there are two main approaches to find a stationary point to the maximum WSR problem. On the one hand, strategies in [2][3] (and references therein) focus on the coordination among transmitters based on the interference-cost concept, where each transmitter

Copyright (c) 2015 IEEE. Personal use of this material is permitted. However, permission to use this material for any other purposes must be obtained from the IEEE by sending a request to pubs-permissions @ieee.org.

Manuscript received January 16, 2015; revised May 18, 2015, July 23, 2015, and September 16, 2015; accepted October 2, 2015.

S. Lagen, A. Agustin, and J. Vidal are with the department of Signal Theory and Communications, Universitat Politècnica de Catalunya, Barcelona, Spain. e-mail: \{sandra.lagen, adrian.agustin, josep.vidal\}@upc.edu

This work has been supported by projects TROPIC FP7 ICT-2011-8318784 (European Commission), DISNET TEC2013-41315-R (Ministerio de Economía y Competitividad, Spanish Government and ERDF funds), 2014SGR-60 (Catalan Government), and FPU12/00828 grant (Ministerio de Educación, Cultura y Deporte, Spanish Government). maximizes its own utility function minus the interference-cost, hence managing interference in a decentralized manner. On the other hand, convergence to a stationary point can be obtained by iteratively minimizing the weighted sum of mean square errors (WMSE), see [4], where transmit filters, receive filters, and weighting matrices are alternatively optimized, being the weighting matrices chosen according to the inherent relation between the achievable rate and the mean square error (MSE) [5]. Decentralized implementations of the WMSE approach are analyzed in [4] and [6]. In [4] it is shown that the WMSE approach and the strategies based on the interferencecost ([2][3]) yield almost the same WSR performance, but with less complexity in the WMSE case. In [6], the WMSE approach and the interference-cost concept are combined and, by exploiting the channel reciprocity property available in time division duplex systems, almost the same WSR results are obtained but with less stringent requirements for channel estimation and information reporting than [2][3][4].

In all these works and related ones it is assumed that transmitted signals are proper (or circularly symmetric complex) Gaussian distributed [7]. Proper Gaussian signaling has been shown to be optimal in terms of capacity for the MIMO point-to-point channel [8], and also for the MIMO broadcast channel (MIMO BC) with dirty paper coding as the capacity achieving strategy [9][10]. However, recent results have shown that optimality of proper Gaussian signals does not necessarily hold for the MIMO BC if the transmit strategy is restricted to widely linear transceivers [11][12]. It is shown there that capacity gains can be obtained by employing improper (or circularly asymmetric complex) Gaussian signaling because, in the absence of non-linear interference cancellation, the MIMO BC becomes interference-limited [13]. The same happens in the MIMO IC, where improper Gaussian signaling through the use of widely linear precoding is able to improve the known achievable rates when interference is treated as Gaussian noise [14][15][16] and is also shown to obtain larger degrees of freedom (DoF), i.e. slope of the sum-rate as a function of the signal-to-noise ratio (SNR) at the high SNR regime, [17][18][19].

The difference between proper and improper Gaussian signaling yields in the characterization of the second order statistics: while proper Gaussian random vectors are fully specified by the covariance matrices under the zero-mean assumption, improper Gaussian random vectors are characterized not only by the covariance matrices but also by the pseudocovariance matrices [20][21]. An effective way to generate and estimate improper Gaussian signals is by using widely linear precoding (WLP) [15] and widely linear estimation (WLE) [20], respectively, instead of the commonly used linear 
precoding (LP) and linear estimation (LE) techniques that maintain the proper Gaussian distribution of the signals. WLE is a generalized concept used in communication channels that encounter improper noise [22] and in systems that transmit improper signal constellations [23]. The later case is already under investigation in 3GPP LTE-A [24], see [25]. Recently, strategies for WLP design in the MIMO BC are analyzed in [12], however, little has been done regarding the coordinated design of WLP at transmitters for the MIMO IC.

There are two main approaches in the recent literature on improper Gaussian signaling and widely linear operations for the MIMO IC [26]: $i$ ) the composite real formulation, whereby real and imaginary parts of the MIMO channel matrix are separated, and $i$ ) the complex-valued formulation. Many works in the literature, e.g. [14][17][19], tackle the single-input singleoutput (SISO) IC with improper Gaussian signaling through the composite real formulation by reformulating the problem into an equivalent double-sized real-valued MIMO IC. This way, most of the approaches already developed for the MIMO IC with proper Gaussian signaling can be applied. Further, this formulation has been used to state why improper Gaussian signaling is better at handling interference (and under which conditions) in the SISO Z-IC (a special IC setting) [27] and in the MIMO Z-IC [28]. Nevertheless, in [15] it is shown that some new insights on the use of improper Gaussian signaling can be gained thanks to the use of the complexvalued formulation. They show that for any given set of covariance matrices the achievable rates for the MIMO IC can be improved with improper Gaussian signaling by optimizing the transmit pseudo-covariance matrices. In this regard, transmit covariance and pseudo-covariance optimization techniques are proposed for the two-user SISO IC in [15] and for the $K$-user multiple-input single-output (MISO) IC in [16], but they are only valid for the single-antenna receiver case.

In this work we address the maximization of the WSR in the general $K$-user MIMO IC and we focus on covering a backwards compatibility-oriented scenario where different types of linear and widely linear transmitters coexist. This heterogeneous scenario is of relevance in legacy cellular networks (as 3GPP LTE-A [24]), where user terminals of different releases coexist and different transmission modes (TM) can be adopted at transmitters [29]. In the downlink transmission, LTE-A considers different TM based on non-codebook-based precoding at the base stations, which have been introduced in different releases [29]: TM7 in release 8, TM8 in release 9, TM9 in release 10, and TM10 in release 11 . Therefore, the heterogeneous transmitter scenario can appear in the downlink due to the use of transmission modes of different releases. In the uplink transmission, where transmitters are the user terminals, the heterogeneous transmitter scenario naturally arises due to the coexistence of user terminals from different releases. To cover said situations, we assume that some transmitters are constrained to employ conventional LP while other transmitters can apply WLP. The scenario with mixed transmitters is shown in Fig. 1. As an example, transmitter 2 (TX 2) is restricted to use LP and the remaining transmitters can adopt WLP if required.

If all transmitters used WLP then the maximum WSR problem could be addressed with the composite real formulation and conventional schemes developed for the MIMO IC could be applied. However, the backwards compatibilityoriented scenario with mixed transmitters cannot be tackled in a straightforward manner with the composite real formulation: working with double-sized matrices and imposing particular structures over transmit filters (which are needed for LP) entails cumbersome formulation. On the contrary, the complexvalued formulation allows facing the problem for transceiver design with mixed (linear and widely linear) transmitters in a unified and more candid way.

In [30] we used the complex-valued formulation to address the minimization of the sum of MSEs in the $K$-user MIMO IC with heterogeneous receivers. In this work, we use the complex-valued formulation to face the maximization of the WSR in the $K$-user MIMO IC with an heterogeneous deployment of transmitters and assuming optimal receivers.

The contributions of this work are:

- The relation between the mutual information and the MSE matrices is derived when the transmitted signal and the interference-plus-noise signal are improper Gaussian distributed.

- The maximum WSR (maxWSR) problem for (widely) linear transceiver design in the most general $K$-user MIMO IC is formulated in such a way to cover a backwards compatibility-oriented scenario.

- An equivalent minimum WMSE (minWMSE) problem is proposed to solve the maxWSR problem thanks to the use of the complex-valued channel model, and closed-form expressions for mixed transceiver design are derived.

- An iterative algorithm with alternate optimization between (widely) linear transmit filters (WLP-LP), widely linear receive filters (WLE), and weighting matrices is presented. Convergence of the algorithm to a stationary point of the maxWSR problem is demonstrated. Decentralized implementations are described.

We show through simulations that the sum-rate is enhanced with the proposed transceiver design (that includes transmit coordination and improper Gaussian signaling) as compared to interference coordination procedures with proper Gaussian signaling. In this regard, simulation results allow identifying the scenarios where the gains are largest.

Finally, it is not worth recalling that the proposed iterative algorithm is not guaranteed to reach the global maximum WSR solution, so the WSR maximization in the $K$-user MIMO IC is still an open problem.

Organization: The rest of the paper is organized as follows. In Section II the system model for the MIMO IC with heterogeneous transmitters is presented, the signal model using the complex-valued channel model is detailed, and the maxWSR problem for mixed transceiver design is formulated. In Section III an equivalent minWMSE problem is proposed and closedform expressions for mixed transceiver design are derived. Then, a centralized algorithm based on alternate optimization is presented and decentralized implementations are detailed. Section IV shows the simulation results. Finally, concluding remarks are included in Section V. 


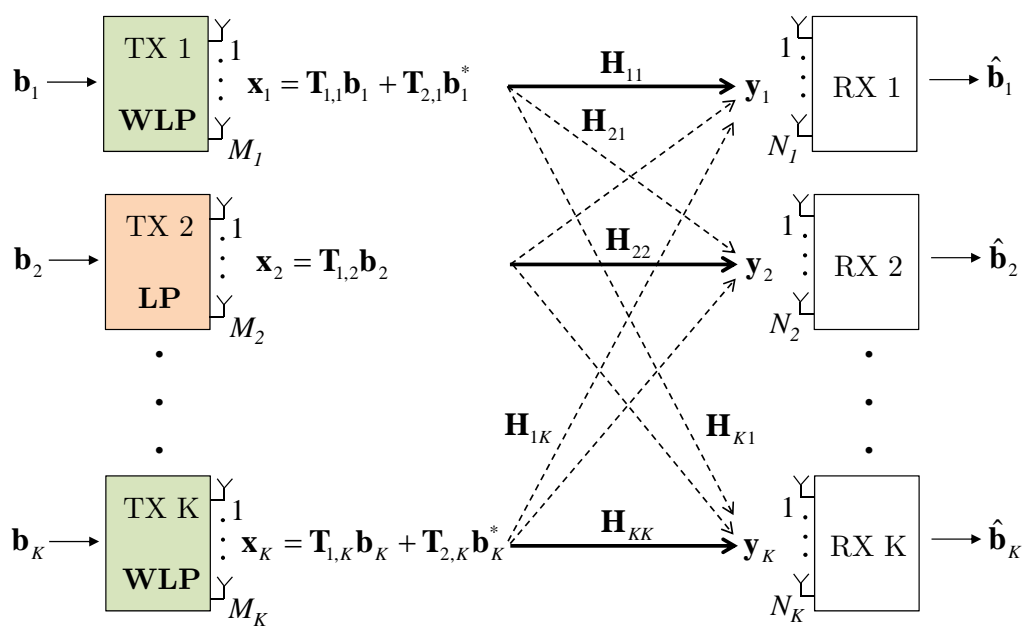

Fig. 1: $K$-user MIMO IC for a backwards compatibility-oriented scenario with mixed transmitters. Solid lines represent desired signals and dashed lines denote interfering signals. In this particular scenario, TX 2 is restricted to employ LP while the other transmitters can adopt WLP if required.

Notation: In this paper, scalars are denoted by italic letters. Boldface lower-case and upper-case letters denote vectors and matrices, respectively. For given scalars $a$ and $b, \min (a, b)$ denotes the minimum between $a$ and $b, \log _{2}(a)$ refers to the base-2 logarithm, $\ln (a)$ to the natural logarithm, and $\lfloor a\rfloor$ to the floor operator. For a given function $f$, the derivative of $f$ with respect to a matrix $\mathbf{A}$ is referred to as $\frac{d f}{d \mathbf{A}}$. For a given matrix $\mathbf{A}, \mathbf{A}^{T}, \mathbf{A}^{*}, \mathbf{A}^{H}$, and $\mathbf{A}^{-1}$ denote the transpose matrix, the conjugate matrix, the hermitian matrix, and the inverse matrix, respectively. The operators $|\mathbf{A}|, \operatorname{Tr}(\mathbf{A}), \mathbb{E}_{\mathbf{x}}[\mathbf{A}]$, and $\Re\{\mathbf{A}\}$ refer to the determinant, the trace, the expectation with respect to variable $\mathbf{x}$, and the real operator, respectively. Matrix I refers to the identity matrix. $\left\{\mathbf{A}_{k}\right\}$ denotes the set of matrices $\left\{\mathbf{A}_{1}, \mathbf{A}_{2}, \ldots, \mathbf{A}_{K}\right\} . \mathbb{C}^{m \times n}$ denotes an $m$ by $n$ dimensional complex space. The circularly symmetric complex normal distribution is represented by $\mathcal{C N}(.,$.$) . For given sets \mathcal{A}$ and $\mathcal{B}$, the intersection is denoted by $\mathcal{A} \cap \mathcal{B}$ and the union is given by $\mathcal{A} \cup \mathcal{B} . \emptyset$ refers to the empty set.

\section{System Model and Problem Formulation}

Consider a MIMO IC composed of a set of $\mathcal{K} \triangleq\{1, \ldots, K\}$ transmitter-receiver pairs, where each $k$-th transmitter is equipped with $M_{k}$ antennas and each $k$-th receiver has $N_{k}$ receive antenna elements $(k=1, \ldots, K)$. On a given time/frequency resource, each $k$-th transmitter serves its associated $k$-th receiver and, therefore, interference is generated among non-associated terminals, as shown in Fig. 1. Assume an heterogeneous scenario of linear and widely linear transmitters (for example, in Fig. 1 TX2 is restricted to use LP while TX1 and TXK can adopt WLP). Define the set of WLP transmitters as $\mathcal{S}^{\mathrm{WLP}}$ and the set of LP transmitters as $\mathcal{S}^{\mathrm{LP}}$, such that $\mathcal{S}^{\mathrm{WLP}} \cap \mathcal{S}^{\mathrm{LP}}=\emptyset$ and $\mathcal{S}^{\mathrm{WLP}} \cup \mathcal{S}^{\mathrm{LP}}=\mathcal{K}$.

\section{A. Signal model}

Without loss of generality, we assume that each $k$-th transmitter intends to send a proper Gaussian information-bearing signal with unitary power towards its intended $k$-th receiver, i.e. $\mathbf{b}_{k} \in \mathbb{C}^{m_{k} \times 1} \sim \mathcal{C N}(\mathbf{0}, \mathbf{I})$, where $m_{k}=\min \left(N_{k}, M_{k}\right)$ denotes the maximum number of streams towards the $k$ th receiver. Unlike conventional transmit linear processing where the transmitted signal is assumed to be proper Gaussian distributed (i.e. $\mathbf{x}_{k} \in \mathbb{C}^{M_{k} \times 1} \sim \mathcal{C N}\left(\mathbf{0}, \mathbf{C}_{\mathbf{x}_{k}}\right)$, being $\mathbf{C}_{\mathbf{x}_{k}}$ the covariance matrix of the transmitted signal), in this paper the general improper Gaussian signaling is used. Hence, the second order statistics of the transmitted signal $\mathbf{x}_{k}$ are not only given by the covariance matrix $\mathbf{C}_{\mathbf{x}_{k}}$ but also by the pseudocovariance matrix $\tilde{\mathbf{C}}_{\mathbf{x}_{k}}$ [20].

Any improper Gaussian signal $\mathbf{x}_{k}$ can be generated from a proper Gaussian information-bearing signal $\mathbf{b}_{k}$ by using WLP (see [15, Sect. II.C]). We assume that legacy transmitters (i.e. $k \in \mathcal{S}^{\mathrm{LP}}$ ) will use the conventional LP scheme. Therefore, the transmitted signal at the $k$-th transmitter is given by:

$$
\mathbf{x}_{k}= \begin{cases}\mathbf{T}_{1, k} \mathbf{b}_{k}+\mathbf{T}_{2, k} \mathbf{b}_{k}^{*} & \text { if } k \in \mathcal{S}^{\mathrm{WLP}} \\ \mathbf{T}_{1, k} \mathbf{b}_{k} & \text { if } k \in \mathcal{S}^{\mathrm{LP}}\end{cases}
$$

where matrices $\mathbf{T}_{1, k}$ and $\mathbf{T}_{2, k} \in \mathbb{C}^{M_{k} \times m_{k}}$ are the transmit linear precoders for the information-bearing signal $\mathbf{b}_{k}$ and its complex conjugate $\mathbf{b}_{k}^{*}$, respectively. This way, the covariance matrix $\mathbf{C}_{\mathbf{x}_{k}}$ and the pseudo-covariance matrix $\tilde{\mathbf{C}}_{\mathbf{x}_{k}}$ of the transmitted signal $\mathbf{x}_{k}$ in (1) result [20]:

$$
\begin{aligned}
\mathbf{C}_{\mathbf{x}_{k}} & =\mathbb{E}_{\mathbf{b}_{k}}\left\{\mathbf{x}_{k} \mathbf{x}_{k}^{H}\right\}=\mathbf{T}_{1, k} \mathbf{T}_{1, k}^{H}+\mathbf{T}_{2, k} \mathbf{T}_{2, k}^{H} \\
\tilde{\mathbf{C}}_{\mathbf{x}_{k}} & =\mathbb{E}_{\mathbf{b}_{k}}\left\{\mathbf{x}_{k} \mathbf{x}_{k}^{T}\right\}=\mathbf{T}_{1, k} \mathbf{T}_{2, k}^{T}+\mathbf{T}_{2, k} \mathbf{T}_{1, k}^{T} .
\end{aligned}
$$

The conventional LP scheme is a special case of WLP in which $\mathbf{T}_{2, k}=\mathbf{0}$ in (1), such that $\mathbf{x}_{k}$ is a proper Gaussian signal (i.e. $\tilde{\mathbf{C}}_{\mathbf{x}_{k}}=\mathbf{0}$ in (3)). The power radiated by the $k$-th transmitter is given by $\operatorname{Tr}\left(\mathbf{C}_{\mathbf{x}_{k}}\right)=\operatorname{Tr}\left(\mathbf{T}_{1, k} \mathbf{T}_{1, k}^{H}+\mathbf{T}_{2, k} \mathbf{T}_{2, k}^{H}\right)$.

Assuming narrow-band transmissions, the equivalent baseband signal observed at the $k$-th receiver is expressed as:

$$
\mathbf{y}_{k}=\mathbf{H}_{k k} \mathbf{x}_{k}+\mathbf{i}_{k}=\mathbf{H}_{k k} \mathbf{x}_{k}+\sum_{j \in \mathcal{K}, j \neq i} \mathbf{H}_{k j} \mathbf{x}_{j}+\mathbf{n}_{k}
$$

where $\mathbf{H}_{k j} \in \mathbb{C}^{N_{k} \times M_{j}}$ denotes the MIMO channel matrix between the $j$-th transmitter and the $k$-th receiver (containing the complex-valued channel gains of the different antennapairs) and $\mathbf{i}_{k} \in \mathbb{C}^{N_{k} \times 1}$ refers to the received interference-plusnoise at the $k$-th receiver. $\mathbf{i}_{k}$ is composed by an interference 
component plus circularly symmetric complex (i.e. proper) Gaussian noise with distribution $\mathbf{n}_{k} \sim \mathcal{C N}\left(\mathbf{0}, \boldsymbol{\Sigma}_{k}\right)$. Hence, under the independence assumption of $\left\{\mathbf{b}_{k}\right\}=\left\{\mathbf{b}_{1}, \ldots, \mathbf{b}_{K}\right\}$ and $\mathbf{n}_{k}$, the covariance matrix $\mathbf{C}_{\mathbf{y}_{k}}$ and the pseudo-covariance matrix $\tilde{\mathbf{C}}_{\mathbf{y}_{k}}$ of the received signal $\mathbf{y}_{k}$ in (4) are:

$$
\begin{aligned}
\mathbf{C}_{\mathbf{y}_{k}}= & \mathbb{E}_{\left\{\mathbf{b}_{k}\right\}, \mathbf{n}_{k}}\left\{\mathbf{y}_{k} \mathbf{y}_{k}^{H}\right\}=\mathbf{H}_{k k} \mathbf{C}_{\mathbf{x}_{k}} \mathbf{H}_{k k}^{H}+\mathbf{C}_{\mathbf{i}_{k}} \\
& \mathbf{C}_{\mathbf{i}_{k}}=\sum_{j \in \mathcal{K}, j \neq i} \mathbf{H}_{k j} \mathbf{C}_{\mathbf{x}_{j}} \mathbf{H}_{k j}^{H}+\boldsymbol{\Sigma}_{k} \\
\tilde{\mathbf{C}}_{\mathbf{y}_{k}}= & \mathbb{E}_{\left\{\mathbf{b}_{k}\right\}, \mathbf{n}_{k}}\left\{\mathbf{y}_{k} \mathbf{y}_{k}^{T}\right\}=\mathbf{H}_{k k} \tilde{\mathbf{C}}_{\mathbf{x}_{k}} \mathbf{H}_{k k}^{T}+\tilde{\mathbf{C}}_{\mathbf{i}_{k}} \\
& \tilde{\mathbf{C}}_{\mathbf{i}_{k}}=\sum_{j \in \mathcal{K}, j \neq i} \mathbf{H}_{k j} \tilde{\mathbf{C}}_{\mathbf{x}_{j}} \mathbf{H}_{k j}^{T}
\end{aligned}
$$

where $\mathbf{C}_{\mathbf{i}_{k}}$ and $\tilde{\mathbf{C}}_{\mathbf{i}_{k}}$ denote the covariance matrix and the pseudo-covariance matrix of the received interference-plusnoise signal $\mathbf{i}_{k}$ in (4), respectively.

\section{B. Widely linear estimation receivers}

In order to access the information contained in the received signal $\mathbf{y}_{k}$ in (4), WLE should be applied at the receiver [20]. Therefore, the information-bearing signal $\mathbf{b}_{k}$ is estimated at the receiver side according to:

$$
\hat{\mathbf{b}}_{k}=\mathbf{R}_{1, k}^{H} \mathbf{y}_{k}+\mathbf{R}_{2, k}^{H} \mathbf{y}_{k}^{*}
$$

where $\mathbf{R}_{1, k}$ and $\mathbf{R}_{2, k} \in \mathbb{C}^{N_{k} \times m_{k}}$ are the linear receive filters.

The MSE for the symbols transmitted towards the $k$-th receiver can be expressed through the so-called MSE-matrix $\mathbf{E}_{k}=\mathbb{E}_{\left\{\mathbf{b}_{k}\right\}, \mathbf{n}_{k}}\left\{\mathbf{e}_{k} \mathbf{e}_{k}^{H}\right\}$, where $\mathbf{e}_{k}=\mathbf{b}_{k}-\hat{\mathbf{b}}_{k}$. As the different information-bearing signals $\left\{\mathbf{b}_{k}\right\}$ correspond to independent and proper Gaussian random vectors (and hence uncorrelated), the MSE-matrix can be developed in terms of the transmit and receive filters in (1) and (7) as:

$$
\begin{aligned}
\mathbf{E}_{k}= & \mathbf{I}-\mathbf{R}_{1, k}^{H} \mathbf{H}_{k k} \mathbf{T}_{1, k}-\mathbf{T}_{1, k}^{H} \mathbf{H}_{k k}^{H} \mathbf{R}_{1, k}-\mathbf{R}_{2, k}^{H} \mathbf{H}_{k k}^{*} \mathbf{T}_{2, k}^{*} \\
& -\mathbf{T}_{2, k}^{T} \mathbf{H}_{k k}^{T} \mathbf{R}_{2, k}+\mathbf{R}_{1, k}^{H} \mathbf{C}_{\mathbf{y}_{k}} \mathbf{R}_{1, k}+\mathbf{R}_{2, k}^{H} \mathbf{C}_{\mathbf{y}_{k}}^{*} \mathbf{R}_{2, k} \\
& +\mathbf{R}_{1, k}^{H} \tilde{\mathbf{C}}_{\mathbf{y}_{k}} \mathbf{R}_{2, k}+\mathbf{R}_{2, k}^{H} \tilde{\mathbf{C}}_{\mathbf{y}_{k}}^{*} \mathbf{R}_{1, k} .
\end{aligned}
$$

Further, an additional matrix is of relevance when working with WLE receivers, which we call the pseudo-MSE-matrix. The pseudo-MSE-matrix is defined as $\tilde{\mathbf{E}}_{k}=\mathbb{E}_{\left\{\mathbf{b}_{k}\right\}, \mathbf{n}_{k}}\left\{\mathbf{e}_{k} \mathbf{e}_{k}^{T}\right\}$, where $\mathbf{e}_{k}=\mathbf{b}_{k}-\hat{\mathbf{b}}_{k}$. Similarly as for the MSE-matrix in (8), we can develop $\tilde{\mathbf{E}}_{k}$ as a function of the transmit and receive filters in (1) and (7):

$$
\begin{aligned}
\tilde{\mathbf{E}}_{k}= & -\mathbf{R}_{1, k}^{H} \mathbf{H}_{k k} \mathbf{T}_{2, k}-\mathbf{T}_{1, k}^{H} \mathbf{H}_{k k}^{H} \mathbf{R}_{2, k}^{*}-\mathbf{R}_{2, k}^{H} \mathbf{H}_{k k}^{*} \mathbf{T}_{1, k}^{*} \\
& -\mathbf{T}_{2, k}^{T} \mathbf{H}_{k k}^{T} \mathbf{R}_{1, k}^{*}+\mathbf{R}_{1, k}^{H} \tilde{\mathbf{C}}_{\mathbf{y}_{k}} \mathbf{R}_{1, k}^{*}+\mathbf{R}_{2, k}^{H} \tilde{\mathbf{C}}_{\mathbf{y}_{k}}^{*} \mathbf{R}_{2, k}^{*} \\
& +\mathbf{R}_{1, k}^{H} \mathbf{C}_{\mathbf{y}_{k}} \mathbf{R}_{2, k}^{*}+\mathbf{R}_{2, k}^{H} \mathbf{C}_{\mathbf{y}_{k}}^{H} \mathbf{R}_{1, k}^{*} .
\end{aligned}
$$

Fixed all the transmit filters, $\left\{\mathbf{T}_{1, k}, \mathbf{T}_{2, k}\right\}$, the optimal widely linear receive filters for the $k$-th receiver are well known in the literature. They are obtained from the minimization of the MSE [20], i.e. $\operatorname{Tr}\left(\mathbf{E}_{k}\right)$, and are given by:

$$
\begin{aligned}
& \mathbf{R}_{1, k}=\mathbf{Q}_{\mathbf{y}_{k}}^{-1}\left(\mathbf{H}_{k k} \mathbf{T}_{1, k}-\tilde{\mathbf{C}}_{\mathbf{y}_{k}} \mathbf{C}_{\mathbf{y}_{k}{ }^{*}} \mathbf{H}_{k k}^{*} \mathbf{T}_{2, k}^{*}\right) \\
& \mathbf{R}_{2, k}=\mathbf{Q}_{\mathbf{y}_{k}}^{-1}\left(\mathbf{H}_{k k}^{*} \mathbf{T}_{2, k}^{*}-\tilde{\mathbf{C}}_{\mathbf{y}_{k}}^{*} \mathbf{C}_{\mathbf{y}_{k}}^{-1} \mathbf{H}_{k k} \mathbf{T}_{1, k}\right)
\end{aligned}
$$

where $\mathbf{Q}_{\mathbf{y}_{k}}=\mathbf{C}_{\mathbf{y}_{k}}-\tilde{\mathbf{C}}_{\mathbf{y}_{k}} \mathbf{C}_{\mathbf{y}_{k}}^{-*} \tilde{\mathbf{C}}_{\mathbf{y}_{k}}^{*}$.
Under the assumption that interference is treated as Gaussian noise, the achievable rate for the $k$-th receiver is given by [15]:

$$
R_{k}=\log _{2} \frac{\left|\mathbf{C}_{\mathbf{y}_{k}}\right|}{\left|\mathbf{C}_{\mathbf{i}_{k}}\right|}+\frac{1}{2} \log _{2} \frac{\left|\mathbf{I}-\mathbf{C}_{\mathbf{y}_{k}}^{-1} \tilde{\mathbf{C}}_{\mathbf{y}_{k}} \mathbf{C}_{\mathbf{y}_{k}}^{-T} \tilde{\mathbf{C}}_{\mathbf{y}_{k}}^{H}\right|}{\left|\mathbf{I}-\mathbf{C}_{\mathbf{i}_{k}}^{-1} \tilde{\mathbf{C}}_{\mathbf{i}_{k}} \mathbf{C}_{\mathbf{i}_{k}}^{-T} \tilde{\mathbf{C}}_{\mathbf{i}_{k}}^{H}\right|} .
$$

Further, it is shown in [28] that the achievable rate $R_{k}$ in (12) is related to the MSE-matrix $\mathbf{E}_{k}$ in (8) and to the pseudoMSE-matrix $\tilde{\mathbf{E}}_{k}$ in (9) through:

$$
R_{k}=\underset{\mathbf{R}_{1, k}, \mathbf{R}_{2, k}}{\operatorname{maximize}}-\frac{1}{2} \log _{2}\left|\mathbf{E}_{k} \mathbf{F}_{k}^{*}\right|
$$

where

$$
\mathbf{F}_{k}=\mathbf{E}_{k}-\tilde{\mathbf{E}}_{k} \mathbf{E}_{k}^{-*} \tilde{\mathbf{E}}_{k}^{*} .
$$

The optimal receive filters $\mathbf{R}_{1, k}, \mathbf{R}_{2, k}$ maximizing the expression in (13) are those in (10)-(11), which can be demonstrated either by using the composite real formulation [20][28] or by plugging the optimal structures of $\mathbf{R}_{1, k}, \mathbf{R}_{1, k}$ in (10)-(11) into $\mathbf{E}_{k}$ in (8) and $\tilde{\mathbf{E}}_{k}$ in (9). Therefore, the receive filters in (10)(11) preserve the achievable rate in (12).

\section{Problem formulation}

When adopting a maximum weighted sum-rate (maxWSR) criterion, the problem of interest is to find (widely) linear transmit filters (or precoders) such that the WSR is maximized while the power budget of each transmitter is respected. Hence, (widely) linear transmit filters $\left\{\mathbf{T}_{1, k}\right\},\left\{\mathbf{T}_{2, k}\right\}$ are obtained as the solution to the following maxWSR problem:

$$
\begin{aligned}
\left(\mathrm{P}_{0}\right): \underset{\left\{\mathbf{T}_{1, k}\right\},\left\{\mathbf{T}_{2, k}\right\}}{\operatorname{maximize}} & \sum_{k \in \mathcal{K}} \mu_{k} R_{k} \\
\text { subject to } & \left\{\begin{array}{l}
\operatorname{Tr}\left(\mathbf{T}_{1, k} \mathbf{T}_{1, k}^{H}+\mathbf{T}_{2, k} \mathbf{T}_{2, k}^{H}\right) \leq P_{k}^{\max } \\
\mathbf{T}_{2, k}=\mathbf{0} \quad \forall k \in \mathcal{S}^{\mathrm{LP}}
\end{array}\right.
\end{aligned}
$$

where $\mu_{k}$ is a weighting coefficient associated to the priority of the $k$-th receiver (thus controlling the quality-of-service of each receiver), $R_{k}$ is the achievable rate for the $k$-th receiver given in (12), and $P_{k}^{\max }$ is the maximum power available at the $k$-th transmitter. The design of the parameter $\mu_{k}$ is not addressed in the paper, but in Section IV-D we analyze an alternative design that allows guaranteeing fairness in the system. The second constraint in (15) imposes $\mathbf{T}_{2, k}=\mathbf{0}$ for those transmitters that are restricted to use LP (i.e. $k \in \mathcal{S}^{\mathrm{LP}}$ ). Due to interference, maxWSR problem $\left(\mathrm{P}_{0}\right)$ in (15) is not convex on $\left\{\mathbf{T}_{1, k}\right\},\left\{\mathbf{T}_{2, k}\right\}$ (either jointly or separately), so the optimal solution cannot be guaranteed.

By using the relation among the achievable rate and the MSE-matrices in (13), the maxWSR problem $\left(\mathrm{P}_{0}\right)$ in (15) can be equivalently written as follows [4, Sect. II.B]:

$$
\begin{aligned}
\left(\hat{\mathrm{P}}_{0}\right): \underset{\substack{\left\{\mathbf{T}_{1, k}\right\},\left\{\mathbf{T}_{2, k}\right\} \\
\left\{\mathbf{R}_{1, k}\right\},\left\{\mathbf{R}_{2, k}\right\}}}{\operatorname{minimize}} & \sum_{k \in \mathcal{K}} \frac{\mu_{k}}{2} \log _{2}\left|\mathbf{E}_{k} \mathbf{F}_{k}^{*}\right| \\
\text { subject to } & \left\{\begin{array}{l}
\operatorname{Tr}\left(\mathbf{T}_{1, k} \mathbf{T}_{1, k}^{H}+\mathbf{T}_{2, k} \mathbf{T}_{2, k}^{H}\right) \leq P_{k}^{\max } \\
\mathbf{T}_{2, k}=\mathbf{0} \quad \forall k \in \mathcal{S}^{\mathrm{LP}}
\end{array}\right.
\end{aligned}
$$

But, again due to interference, the maxWSR problem $\left(\hat{\mathrm{P}}_{0}\right)$ in (16) is not convex on $\left\{\mathbf{T}_{1, k}\right\},\left\{\mathbf{T}_{2, k}\right\},\left\{\mathbf{R}_{1, k}\right\},\left\{\mathbf{R}_{2, k}\right\}$. 


$$
\begin{aligned}
\left(\mathrm{P}_{1}\right): \underset{\substack{\left\{\mathbf{T}_{1, k}\right\},\left\{\mathbf{T}_{2, k}\right\} \\
\left\{\mathbf{R}_{1, k},\left\{\mathbf{R}_{2, k}\right\} \\
\left\{\mathbf{W}_{1, k}\right\},\left\{\mathbf{W}_{2, k}\right\}\right.}}{\operatorname{minimize}} & \sum_{k \in \mathcal{K}}\left(\frac{1}{2} \operatorname{Tr}\left(\mathbf{W}_{1, k} \mathbf{E}_{k}+\mathbf{W}_{1, k}^{*} \mathbf{E}_{k}^{*}+\mathbf{W}_{2, k}^{*} \tilde{\mathbf{E}}_{k}+\mathbf{W}_{2, k} \tilde{\mathbf{E}}_{k}^{*}\right)-\frac{\mu_{k}}{2} \log _{2}\left|\frac{\ln (2)}{\mu_{k}} \overline{\mathbf{W}}_{k}\right|\right) \\
& \text { subject to } \quad\left\{\begin{array}{l}
\operatorname{Tr}\left(\mathbf{T}_{1, k} \mathbf{T}_{1, k}^{H}+\mathbf{T}_{2, k} \mathbf{T}_{2, k}^{H}\right) \leq P_{k}^{\max } \forall k \\
\mathbf{T}_{2, k}=\mathbf{0} \quad \forall k \in \mathcal{S}^{\mathrm{LP}}
\end{array}\right.
\end{aligned}
$$

The equivalence among the maxWSR problems $\left(\mathrm{P}_{0}\right)$ in $(15)$ and $\left(\hat{\mathrm{P}}_{0}\right)$ in $(16)$ is in the sense that the global optimal solution $\left\{\mathbf{T}_{1, k}^{\star}, \mathbf{T}_{2, k}^{\star}\right\}$ for the two problems is identical. Further, if $\left\{\mathbf{T}_{1, k}^{\star}, \mathbf{T}_{2, k}^{\star}, \mathbf{R}_{1, k}^{\star}, \mathbf{R}_{2, k}^{\star}\right\}$ is a stationary point of $\left(\hat{\mathrm{P}}_{0}\right)$ then $\left\{\mathbf{T}_{1, k}^{\star}, \mathbf{T}_{2, k}^{\star}\right\}$ is a stationary point of $\left(\mathrm{P}_{0}\right)$ (and the converse).

\section{EQUIVALENT WMSE FORMULATION}

In this section we propose an equivalent minimum weighted mean square error (minWMSE) problem that will allow tackling the maxWSR problem $\left(\hat{\mathrm{P}}_{0}\right)$ presented in (16) for the case of improper Gaussian signaling with mixed transceivers.

Let us introduce auxiliary weighting matrices $\mathbf{W}_{1, k}$ and $\mathbf{W}_{2, k}$ for the $k$-th receiver, being $\mathbf{W}_{1, k}$ an hermitian positive semidefinite matrix and $\mathbf{W}_{2, k}$ a symmetric matrix. Then, the following result establishes the equivalence between the maxWSR problem $\left(\hat{\mathrm{P}}_{0}\right)$ in $(16)$ and the minWMSE problem $\left(\mathrm{P}_{1}\right)$ proposed in (17).

Theorem 1: The maxWSR problem $\left(\hat{\mathrm{P}}_{0}\right)$ in $(16)$ is equivalent to the minWMSE problem $\left(\mathrm{P}_{1}\right)$ shown in (17) at the top of the page, where $\mathbf{E}_{k}$ and $\tilde{\mathbf{E}}_{k}$ correspond to the MSE-matrix and the pseudo-MSE-matrix for the $k$-th receiver detailed in (8) and (9), respectively, and $\overline{\mathbf{W}}_{k}$ is a block matrix given by:

$$
\overline{\mathbf{W}}_{k}=\left[\begin{array}{ll}
\mathbf{W}_{1, k} & \mathbf{W}_{2, k} \\
\mathbf{W}_{2, k}^{*} & \mathbf{W}_{1, k}^{*}
\end{array}\right]
$$

which is hermitian positive semidefinite by construction.

The equivalence among the maxWSR problem $\left(\hat{\mathrm{P}}_{0}\right)$ in (16) and the minWMSE problem $\left(\mathrm{P}_{1}\right)$ in (17) is in the sense that the global optimal solution $\left\{\mathbf{T}_{1, k}^{\star}, \mathbf{T}_{2, k}^{\star}, \mathbf{R}_{1, k}^{\star}, \mathbf{R}_{2, k}^{\star}\right\}$ for the two problems is identical. Furthermore, if $\left\{\mathbf{T}_{1, k}^{\star}, \mathbf{T}_{2, k}^{\star}, \mathbf{R}_{1, k}^{\star}, \mathbf{R}_{2, k}^{\star}, \mathbf{W}_{1, k}^{\star}, \mathbf{W}_{2, k}^{\star}\right\}$ is a stationary point of $\left(\mathbf{P}_{1}\right)$ then $\left\{\mathbf{T}_{1, k}^{\star}, \mathbf{T}_{2, k}^{\star}, \mathbf{R}_{1, k}^{\star}, \mathbf{R}_{2, k}^{\star}\right\}$ is a stationary point of $\left(\hat{\mathrm{P}}_{0}\right)$ (and the converse), as they satisfy the first-order optimality conditions of both problems.

Proof: See Appendix A.

The advantage of $\left(\mathrm{P}_{1}\right)$ in (17) as compared to $\left(\mathrm{P}_{0}\right)$ in (15) and $\left(\hat{\mathrm{P}}_{0}\right)$ in (16) is that it is convex on each set of variables separately (i.e. it is convex on $\left\{\mathbf{T}_{1, k}\right\}$ given $\left\{\mathbf{T}_{2, k}\right\},\left\{\mathbf{R}_{1, k}\right\}$, $\left\{\mathbf{R}_{2, k}\right\},\left\{\mathbf{W}_{1, k}\right\}$, and $\left\{\mathbf{W}_{2, k}\right\}$, and so on). This property suggests that a block coordinate descent (BCD) method [31] could be used to find a stationary point to $\left(\mathrm{P}_{1}\right)$ in (17).

Let us emphasize that problem $\left(\mathrm{P}_{1}\right)$ in (17) is also valid to cover the minimization of the sum of MSE in the $K$-user MIMO IC if we set $\mathbf{W}_{2, k}=\mathbf{0}$ and $\mathbf{W}_{1, k}=\mathbf{I}, \forall k$, such that the objective function in (17) simply results: $\sum_{k \in \mathcal{K}} \operatorname{Tr}\left(\mathbf{E}_{k}\right)$. In this case, the use of heterogeneous (linear and widely linear) receivers could be included into the problem formulation, see [30], as the use of LE or WLE affects the MSE but might not impact on the maximum achievable rate.

\section{A. Alternate optimization}

In this section we exploit the convex properties of the minWMSE problem $\left(\mathrm{P}_{1}\right)$ in (17) so as to find a stationary point solution. First, by checking the first-order optimality conditions of $\left(\mathrm{P}_{1}\right)$ in (17) and manipulating the obtained equalities, we derive analytical expressions for each paired-set of variables (i.e. $\left\{\mathbf{R}_{1, k}, \mathbf{R}_{2, k}\right\},\left\{\mathbf{W}_{1, k}, \mathbf{W}_{2, k}\right\}$, and $\left\{\mathbf{T}_{1, k}, \mathbf{T}_{2, k}\right\}$ ) assuming that the remaining paired-sets are fixed. The results are given in the following propositions. Then, in Section III-B we propose an algorithm based on the BCD method with alternate optimization among the paired-sets of variables that is shown to reach a stationary point to $\left(\mathrm{P}_{1}\right)$ in (17).

The optimal widely linear receive filters $\left\{\mathbf{R}_{1, k}, \mathbf{R}_{2, k}\right\}$ to the maxWSR problem $\left(\hat{\mathrm{P}}_{0}\right)$ in (16) for given transmit filters $\left\{\mathbf{T}_{1, k}, \mathbf{T}_{2, k}\right\}$ are those in (10)-(11), as the objective function can be uncoupled for each receiver when considering the variables $\left\{\mathbf{R}_{1, k}, \mathbf{R}_{2, k}\right\}$ and the receive filters in (10)-(11) are known to preserve the achievable rate (see (13)). Accordingly, $\left\{\mathbf{R}_{1, k}, \mathbf{R}_{2, k}\right\}$ in (10)-(11) are the optimal widely linear receive filters to the minWMSE problem $\left(\hat{\mathrm{P}}_{1}\right)$ in (17) for given transmit filters $\left\{\mathbf{T}_{1, k}, \mathbf{T}_{2, k}\right\}$ and weighting matrices $\left\{\mathbf{W}_{1, k}, \mathbf{W}_{2, k}\right\}$.

The result for the optimal weighting matrices $\left\{\mathbf{W}_{1, k}, \mathbf{W}_{2, k}\right\}$ when $\left\{\mathbf{T}_{1, k}, \mathbf{T}_{2, k}\right\}$ and $\left\{\mathbf{R}_{1, k}, \mathbf{R}_{2, k}\right\}$ are fixed is detailed in the following proposition.

Proposition 1: For given transmit filters $\left\{\mathbf{T}_{1, k}, \mathbf{T}_{2, k}\right\}$ and receive filters $\left\{\mathbf{R}_{1, k}, \mathbf{R}_{2, k}\right\}$, the optimal weighting matrices $\left\{\mathbf{W}_{1, k}, \mathbf{W}_{2, k}\right\}$ to the minWMSE problem $\left(\mathrm{P}_{1}\right)$ in (17) are:

$$
\begin{aligned}
\mathbf{W}_{1, k} & =\frac{\mu_{k}}{\ln (2)} \mathbf{F}_{k}^{-1} \\
\mathbf{W}_{2, k} & =-\frac{\mu_{k}}{\ln (2)} \mathbf{E}_{k}^{-1} \tilde{\mathbf{E}}_{k} \mathbf{F}_{k}^{-*}
\end{aligned}
$$

where $\mathbf{F}_{k}$ is the one defined in (14).

Proof: See Appendix A.

In order to compact the (widely) linear transmit filter design, let us define the following matrices:

$$
\begin{aligned}
\mathbf{A}_{k} & =\mathbf{H}_{k k}^{H}\left(\mathbf{R}_{1, k} \mathbf{W}_{1, k} \mathbf{R}_{1, k}^{H}+\mathbf{R}_{2, k}^{*} \mathbf{W}_{1, k}^{*} \mathbf{R}_{2, k}^{T}\right) \mathbf{H}_{k k}+\mathbf{\Upsilon}_{k} \\
\boldsymbol{\Upsilon}_{k} & =\sum_{j=1, j \neq k}^{K} \mathbf{H}_{j k}^{H}\left(\mathbf{R}_{1, j} \mathbf{W}_{1, j} \mathbf{R}_{1, j}^{H}+\mathbf{R}_{2, j}^{*} \mathbf{W}_{1, j}^{*} \mathbf{R}_{2, j}^{T}\right) \mathbf{H}_{j k} \\
\mathbf{B}_{k} & =\mathbf{H}_{k k}^{H}\left(\mathbf{R}_{1, k} \mathbf{W}_{1, k} \mathbf{R}_{2, k}^{H}+\mathbf{R}_{2, k}^{*} \mathbf{W}_{1, k}^{*} \mathbf{R}_{1, k}^{T}\right) \mathbf{H}_{k k}^{*}+\boldsymbol{\Gamma}_{k} \\
\boldsymbol{\Gamma}_{k} & =\sum_{j=1, j \neq k}^{K} \mathbf{H}_{j k}^{H}\left(\mathbf{R}_{1, j} \mathbf{W}_{1, j} \mathbf{R}_{2, j}^{H}+\mathbf{R}_{2, j}^{*} \mathbf{W}_{1, j}^{*} \mathbf{R}_{1, j}^{T}\right) \mathbf{H}_{j k}^{*} \\
\mathbf{C}_{k} & =\mathbf{H}_{k k}^{H}\left(\mathbf{R}_{1, k} \mathbf{W}_{2, k} \mathbf{R}_{2, k}^{T}+\mathbf{R}_{2, k}^{*} \mathbf{W}_{2, k}^{*} \mathbf{R}_{1, k}^{H}\right) \mathbf{H}_{k k}+\boldsymbol{\Phi}_{k} \\
\boldsymbol{\Phi}_{k} & =\sum_{j=1, j \neq k}^{K} \mathbf{H}_{j k}^{H}\left(\mathbf{R}_{1, j} \mathbf{W}_{2, j} \mathbf{R}_{2, j}^{T}+\mathbf{R}_{2, j}^{*} \mathbf{W}_{2, j}^{*} \mathbf{R}_{1, j}^{H}\right) \mathbf{H}_{j k}
\end{aligned}
$$




$$
\begin{aligned}
& \mathbf{T}_{1, k}= \begin{cases}\mathbf{G}_{k}^{-1}\left(\mathbf{H}_{k k}^{H} \mathbf{R}_{1, k} \mathbf{W}_{1, k}+\mathbf{H}_{k k}^{H} \mathbf{R}_{2, k}^{*} \mathbf{W}_{2, k}^{*}-\mathbf{J}_{k}\left(\mathbf{H}_{k k}^{T} \mathbf{R}_{2, k} \mathbf{W}_{1, k}+\mathbf{H}_{k k}^{T} \mathbf{R}_{1, k}^{*} \mathbf{W}_{2, k}^{*}\right)\right) & \forall k \in \mathcal{S}^{\mathrm{WLP}} \\
\left(\mathbf{A}_{k}+\mathbf{C}_{k}+\lambda_{k} \mathbf{I}\right)^{-1}\left(\mathbf{H}_{k k}^{H} \mathbf{R}_{1, k} \mathbf{W}_{1, k}+\mathbf{H}_{k k}^{H} \mathbf{R}_{2, k}^{*} \mathbf{W}_{2, k}^{*}\right) & \forall k \in \mathcal{S}^{\mathrm{LP}}\end{cases} \\
& \mathbf{T}_{2, k}= \begin{cases}\mathbf{G}_{k}^{-1}\left(\mathbf{H}_{k k}^{H} \mathbf{R}_{2, k}^{*} \mathbf{W}_{1, k}^{*}+\mathbf{H}_{k k}^{H} \mathbf{R}_{1, k} \mathbf{W}_{2, k}-\mathbf{J}_{k}\left(\mathbf{H}_{k k}^{T} \mathbf{R}_{1, k}^{*} \mathbf{W}_{1, k}^{*}+\mathbf{H}_{k k}^{T} \mathbf{R}_{2, k} \mathbf{W}_{2, k}\right)\right) & \forall k \in \mathcal{S}^{\mathrm{WLP}} \\
\mathbf{0} & \forall k \in \mathcal{S}^{\mathrm{LP}}\end{cases}
\end{aligned}
$$

$$
\begin{aligned}
\mathbf{D}_{k} & =\mathbf{H}_{k k}^{H}\left(\mathbf{R}_{1, k} \mathbf{W}_{2, k} \mathbf{R}_{1, k}^{T}+\mathbf{R}_{2, k}^{*} \mathbf{W}_{2, k}^{*} \mathbf{R}_{2, k}^{H}\right) \mathbf{H}_{k k}^{*}+\mathbf{\Psi}_{k} \\
\boldsymbol{\Psi}_{k} & =\sum_{j=1, j \neq k}^{K} \mathbf{H}_{j k}^{H}\left(\mathbf{R}_{1, j} \mathbf{W}_{2, j} \mathbf{R}_{1, j}^{T}+\mathbf{R}_{2, j}^{*} \mathbf{W}_{2, j}^{*} \mathbf{R}_{2, j}^{H}\right) \mathbf{H}_{j k}^{*} .
\end{aligned}
$$

The optimal (widely) linear transmit filters $\left\{\mathbf{T}_{1, k}, \mathbf{T}_{2, k}\right\}$ for fixed $\left\{\mathbf{R}_{1, k}, \mathbf{R}_{2, k}\right\}$ and $\left\{\mathbf{W}_{1, k}, \mathbf{W}_{2, k}\right\}$ are detailed in the following proposition.

Proposition 2: For given receive filters $\left\{\mathbf{R}_{1, k}, \mathbf{R}_{2, k}\right\}$ and weighting matrices $\left\{\mathbf{W}_{1, k}, \mathbf{W}_{2, k}\right\}$, the optimal transmit filters $\left\{\mathbf{T}_{1, k}, \mathbf{T}_{2, k}\right\}$ to the minWMSE problem $\left(\mathrm{P}_{1}\right)$ in (17) are given by equations (29) and (30) at the top of the page, where:

$$
\begin{aligned}
\mathbf{G}_{k}= & \mathbf{A}_{k}+\mathbf{C}_{k}+\lambda_{k} \mathbf{I} \\
& -\left(\mathbf{B}_{k}+\mathbf{D}_{k}\right)\left(\mathbf{A}_{k}+\mathbf{C}_{k}+\lambda_{k} \mathbf{I}\right)^{-*}\left(\mathbf{B}_{k}^{*}+\mathbf{D}_{k}^{*}\right) \\
\mathbf{J}_{k}= & \left(\mathbf{B}_{k}+\mathbf{D}_{k}\right)\left(\mathbf{A}_{k}+\mathbf{C}_{k}+\lambda_{k} \mathbf{I}\right)^{-*}
\end{aligned}
$$

and $\lambda_{k}$ is a non-negative dual variable associated to the $k$ th transmit power constraint in (17). Since $\left(\mathbf{A}_{k}+\mathbf{C}_{k}+\lambda_{k} \mathbf{I}\right)$ is a full rank matrix, it can be proven by means of the Schur complement property [32] that the inverse of $\mathbf{G}_{k}$ in (31) exists.

Proof: See Appendix B.

\section{B. Algorithm}

In order to solve $\left(\mathrm{P}_{1}\right)$ in (17) we use the block coordinate descent (BCD) method [31] with alternate optimization among the three different paired-sets of variables (i.e. $\left\{\mathbf{R}_{1, k}, \mathbf{R}_{2, k}\right\}$, $\left\{\mathbf{W}_{1, k}, \mathbf{W}_{2, k}\right\}$, and $\left.\left\{\mathbf{T}_{1, k}, \mathbf{T}_{2, k}\right\}\right)$. The alternate optimization is detailed in Algorithm 1. By departing from an initialization of the (widely) linear transmit filters that satisfies the pertransmitter power constraints in (17), then we alternatively update: $i)$ receive filters $\left\{\mathbf{R}_{1, k}, \mathbf{R}_{2, k}\right\}$ using (10)-(11), ii) weighting matrices $\left\{\mathbf{W}_{1, k}, \mathbf{W}_{2, k}\right\}$ using (19)-(20), and iii) transmit filters $\left\{\mathbf{T}_{1, k}, \mathbf{T}_{2, k}\right\}$ using (29)-(30). The procedure is iterated until a stop condition (e.g. convergence is achieved, tolerance criterion is met, or maximum number of iterations is reached).

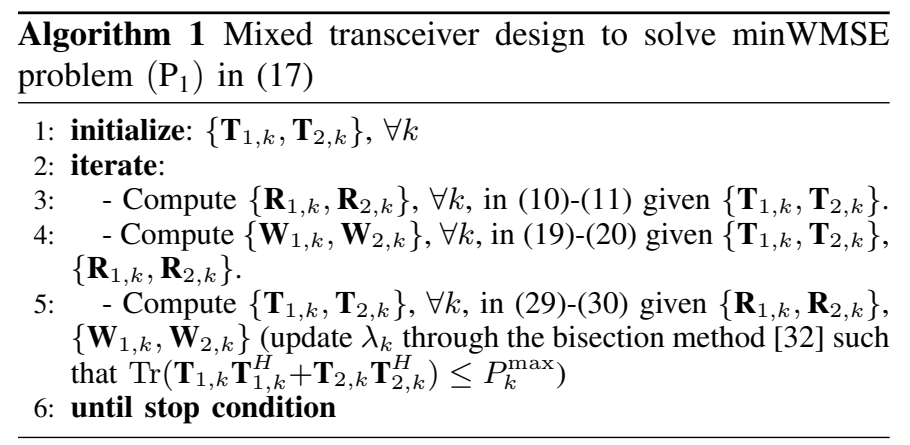

Note that if all transmit filters were initialized with LP (i.e. $\mathbf{T}_{2, k}=\mathbf{0}, \forall k$ ), then all receivers would get $\mathbf{R}_{2, k}=\mathbf{0}$ in (11) since $\mathbf{T}_{2, k}=\mathbf{0}$ and $\tilde{\mathbf{C}}_{\mathbf{y}_{k}}=\mathbf{0}, \forall k$, and the proposed iterative procedure would get stuck to a LP-LE scheme (i.e. properbased solution). A similar observation was found in [12] with iterative algorithms for the MIMO BC: it was shown that a WLP initialization was required to get an improper-based solution. In Algorithm 1, so as to avoid getting stuck to a proper-based solution, it is required that at least one transmitter is initialized with WLP.

The procedure detailed in Algorithm 1 can be implemented either in a centralized or decentralized manner. In the centralized implementation, a central processing node gathers all the channel matrices in the system (i.e. $\mathbf{H}_{k j}, \forall k, \forall j$ ) and then performs the iterative procedure in Algorithm 1. After that, transmitters are informed of the transmit filters to be used and each receiver updates its receive filters based on the received signal and an estimation of the equivalent channel (as it is done in practical implementations [33][25]). On the other hand, in the decentralized implementation, the alternate optimization in Algorithm 1 is combined among transmitters and receivers: receivers perform lines 3 and 4 (i.e. update of receive filters and weighting matrices), while transmitters execute line 5 (i.e. update of transmit filters). To carry out such updates it is required that each $k$-th transmitter has available the channel matrices towards all receivers in the network (i.e. $\mathbf{H}_{j k}, \forall j$ ). Further, it is also required that each receiver has an additional link to feedback the updated receive filters and weighting matrices to all transmitters in the network at each iteration (similar as in the linear transceiver case in [4]). In order to avoid such large requirements for channel knowledge and feedback links, in Section III-E we describe how the minWMSE problem $\left(\mathrm{P}_{1}\right)$ in (17) could be decomposed to obtain alternative decentralized procedures.

If Algorithm 1 is implemented in a central processing node, then LP transmitters do not need to be aware that improper Gaussian signaling is being used in the network. However, for decentralized implementations it is required that LP transmitters know that WLE is being applied at receivers.

\section{Convergence}

The minWMSE problem $\left(\mathrm{P}_{1}\right)$ in (17) is convex on each set of variables separately and closed-form expressions have been derived. This ensures that if we update the sets of variables one-by-one then a monotonic reduction of the objective function of $\left(\mathrm{P}_{1}\right)$ is obtained. Further, by checking the first-order optimality conditions we have arrived to a unique optimal solution for each paired-set of variables given the other two paired-sets of variables (e.g. $\left\{\mathbf{T}_{1, k}, \mathbf{T}_{2, k}\right\}$ given $\left\{\mathbf{R}_{1, k}, \mathbf{R}_{2, k}\right\}$ and $\left.\left\{\mathbf{W}_{1, k}, \mathbf{W}_{2, k}\right\}\right)$. This way, the two sets on each paired-set can be updated simultaneously while maintaining the monotonic reduction properties of the BCD method applied over the 
minWMSE problem $\left(\mathrm{P}_{1}\right)$. Accordingly, with the alternating minimization process in Algorithm 1, the objective function of $\left(\mathrm{P}_{1}\right)$ in (17) decreases monotonically at each iteration.

Theorem 2: Algorithm 1 converges to a stationary point of the maxWSR problem $\left(\mathrm{P}_{0}\right)$ in (15).

Proof: Due to the equivalent relationships among the maxWSR problems $\left(\mathrm{P}_{0}\right)$ in $(15)$ and $\left(\hat{\mathrm{P}}_{0}\right)$ in $(16)$, and among the maxWSR problem $\left(\hat{\mathrm{P}}_{0}\right)$ in $(16)$ and the minWMSE problem $\left(\mathrm{P}_{1}\right)$ in (17), it is sufficient to show that Algorithm 1 converges to a stationary point of $\left(\mathrm{P}_{1}\right)$. When we fix two of the paired-sets of variables (e.g. $\left\{\mathbf{R}_{1, k}, \mathbf{R}_{2, k}\right\}$ and $\left\{\mathbf{W}_{1, k}, \mathbf{W}_{2, k}\right\}$ ), a unique optimal solution is obtained for the remaining pairedset of variables (e.g. $\left\{\mathbf{T}_{1, k}, \mathbf{T}_{2, k}\right\}$ ). Accordingly, the alternate optimization in Algorithm 1 ensures a monotonic reduction of the objective function of $\left(\mathrm{P}_{1}\right)$ at each iteration and, as the objective function is bounded by the optimal value, convergence in terms of the objective function is achieved. Furthermore, since $\left(\mathrm{P}_{1}\right)$ has a differentiable objective function, the constraint set is separable among the paired-sets of variables, and the problem has a unique minimum point along any coordinate direction, then it follows from general optimization theory (see [34, Sect. 8.9]) that Algorithm I (that is based on the BCD method) converges to a stationary point of $\left(\mathrm{P}_{1}\right)$. Finally, due to Theorem 1, the obtained solution is also a stationary point of the maxWSR problem $\left(\hat{\mathrm{P}}_{0}\right)$ in (16) (and, equivalently, of $\left(\mathrm{P}_{0}\right)$ in $\left.(15)\right)$.

\section{Complexity}

The complexity of the proposed Algorithm 1 is related to the number of iterations. Simulations show that around 15-20 iterations are enough to reach a stationary point of the maxWSR problem. However, the larger improvement is obtained in the first iterations (see Section IV-A). For decentralized implementations, the number of iterations is relevant and should be limited. In this case, Theorem 2 demonstrates that at each iteration the objective function of $\left(\mathrm{P}_{1}\right)$ in (17) is monotonically reduced, which in the general case leads to an improvement of the objective function of $\left(\mathrm{P}_{0}\right)$ in (15) (although a monotonic WSR improvement cannot be guaranteed). It is important to recall that the proposed Algorithm 1 already outperforms the conventional coordinated linear transceiver schemes with a low number of iterations (e.g. 5, see Section IV-A).

At each iteration of Algorithm 1, three different updates are performed (i.e. one update for each paired-set of variables). For simplicity of the complexity analysis, let $K$ be the total number of transmit-receive pairs, let $M, N$ denote the number of antennas at each transmitter and receiver, respectively, and let $m$ be the number of streams per receiver. We assume that all transceivers are widely linear to compute an upper bound of the total complexity. For matrices $\mathbf{X} \in \mathbb{C}^{a \times b}, \mathbf{Y} \in \mathbb{C}^{b \times c}$, $\mathbf{Z} \in \mathbb{C}^{d \times d}$, the complexity of the product $\mathbf{X Y}$ is $\mathcal{O}(a b c)$, the complexity of the sum $\mathbf{X}+\mathbf{X}$ is $\mathcal{O}(a b)$, and the complexity of the inverse $\mathbf{Z}^{-1}$ is $\mathcal{O}\left(d^{3}\right)$ [35]. Then, the complexity at each step in one iteration of Algorithm 1 is:

- updating the receive filters for all receivers (line 3): $\mathcal{O}\left(K^{2} M N m+K^{2} N^{2} m+K N^{3}\right)$,

- updating the weighting matrices for all receivers (line 4): $\mathcal{O}\left(K N^{2} m+K m^{2} N+K m^{3}\right)$,
- updating the transmit filters for all receivers (line 5): $\mathcal{O}\left(K^{2} M N m+K^{2} M^{2} m+K^{2} M m^{2}+K M^{3}\right)$.

If we use the upper bound $m<M$, the total complexity per iteration of the proposed Algorithm 1 is upper bounded by: $\mathcal{O}\left(K^{2} M^{3}+K^{2} M^{2} N+K^{2} N^{2} M+K N^{3}\right)$. The order of complexity is the same as the one associated to the algorithm proposed in [4] for the linear transceiver case.

\section{E. Decentralized formulation}

By taking into account the decompositions of matrices $\mathbf{A}_{k}$ in (21), $\mathbf{B}_{k}$ in (23), $\mathbf{C}_{k}$ in (25), and $\mathbf{D}_{k}$ in (27), decentralized approaches could be derived if each $k$-th transmitter had knowledge of $\boldsymbol{\Upsilon}_{k}$ in (22), $\boldsymbol{\Gamma}_{k}$ in (24), $\boldsymbol{\Phi}_{k}$ in (26), and $\boldsymbol{\Psi}_{k}$ in (28), $\mathbf{C}_{\mathbf{i}_{k}}$ in (5), and $\tilde{\mathbf{C}}_{\mathbf{i}_{k}}$ in (6). $\boldsymbol{\Upsilon}_{k}, \boldsymbol{\Gamma}_{k}, \boldsymbol{\Phi}_{k}$, and $\boldsymbol{\Psi}_{k}$ are the terms that require knowledge of information not available either at the $k$-th transmitter or at the $k$-th receiver, while $\mathbf{C}_{\mathbf{i}_{k}}$ and $\tilde{\mathbf{C}}_{\mathbf{i}_{k}}$ could be reported by the intended $k$-th receiver.

Proposition 3: The minWMSE problem $\left(\mathrm{P}_{1}\right)$ in (17) can be decomposed into $K$ parallel optimization problems (one per transmitter). The problem to be solved at the $k$-th transmitter for a fixed $\boldsymbol{\Upsilon}_{k}, \boldsymbol{\Gamma}_{k}, \boldsymbol{\Phi}_{k}, \boldsymbol{\Psi}_{k}, \mathbf{C}_{\mathbf{i}_{k}}$, and $\tilde{\mathbf{C}}_{\mathbf{i}_{k}}$, is:

$$
\begin{aligned}
& \left(\mathrm{P}_{1}^{k}\right): \underset{\mathbf{T}_{1, k}, \mathbf{T}_{2, k}}{\operatorname{minimize}} f_{k}+g_{k} \\
& \mathbf{R}_{1, k}, \mathbf{T}_{2, k} \\
& \begin{array}{c}
\mathbf{R}_{1, k}, \mathbf{R}_{2, k} \\
\mathbf{W}_{1, k}, \mathbf{W}_{2, k}
\end{array} \\
& \text { subject to }\left\{\begin{array}{l}
\operatorname{Tr}\left(\mathbf{T}_{1, k} \mathbf{T}_{1, k}^{H}+\mathbf{T}_{2, k} \mathbf{T}_{2, k}^{H}\right) \leq P_{k}^{\max } \\
\mathbf{T}_{2, k}=\mathbf{0} \quad \forall k \in \mathcal{S}^{\mathrm{LP}}
\end{array}\right.
\end{aligned}
$$

where $f_{k}$ accounts for the impact over the intended receiver:

$$
\begin{aligned}
f_{k}= & \frac{1}{2} \operatorname{Tr}\left(\mathbf{W}_{1, k} \mathbf{E}_{k}+\mathbf{W}_{1, k}^{*} \mathbf{E}_{k}^{*}+\mathbf{W}_{2, k}^{*} \tilde{\mathbf{E}}_{k}+\mathbf{W}_{2, k} \tilde{\mathbf{E}}_{k}^{*}\right) \\
& -\frac{\mu_{k}}{2} \log _{2}\left|\frac{\ln (2)}{\mu_{k}} \overline{\mathbf{W}}_{k}\right|
\end{aligned}
$$

while $g_{k}$ reflects the impact over the unintended receivers:

$$
\begin{aligned}
g_{k}= & \operatorname{Tr}\left(\left(\boldsymbol{\Upsilon}_{k}+\boldsymbol{\Phi}_{k}\right)\left(\mathbf{T}_{1, k} \mathbf{T}_{1, k}^{H}+\mathbf{T}_{2, k} \mathbf{T}_{2, k}^{H}\right)\right) \\
& +\operatorname{Tr}\left(\left(\boldsymbol{\Gamma}_{k}+\mathbf{\Psi}_{k}\right)\left(\mathbf{T}_{2, k}^{*} \mathbf{T}_{1, k}^{H}+\mathbf{T}_{1, k} \mathbf{T}_{2, k}^{T}\right)\right) .
\end{aligned}
$$

Proof: The first-order optimality conditions of the minWMSE problem $\left(\mathrm{P}_{1}\right)$ in (17) and the decomposed problem $\left(\mathrm{P}_{1}^{k}\right)$ in (33) are the same for a fixed $\boldsymbol{\Upsilon}_{k}, \boldsymbol{\Gamma}_{k}, \boldsymbol{\Phi}_{k}, \mathbf{\Psi}_{k}, \mathbf{C}_{\mathbf{i}_{k}}$, and $\tilde{\mathbf{C}}_{\mathbf{i}_{k}}$. See the conditions in Appendix A and B.

Problem $\left(\mathrm{P}_{1}^{k}\right)$ in (33) is convex on each variable separately and it leads to solutions in (10)-(11), (19)-(20), and (29)-(30). Then, each $k$-th transmitter can solve problem $\left(\mathrm{P}_{1}^{k}\right)$ in (33) with alternate optimization between receive filters $\mathbf{R}_{1, k}, \mathbf{R}_{2, k}$, weighting matrices $\mathbf{W}_{1, k}, \mathbf{W}_{2, k}$, and transmit filters $\mathbf{T}_{1, k}, \mathbf{T}_{2, k}$.

A possible mechanism to obtain the matrices $\mathbf{\Upsilon}_{k}, \boldsymbol{\Phi}_{k}$, $\boldsymbol{\Gamma}_{k}$, and $\boldsymbol{\Psi}_{k}$ (also called the interference-cost and pseudointerference cost matrices [30]) is by exchanging controlplane messages among transmitters, as $\boldsymbol{\Upsilon}_{k}, \boldsymbol{\Phi}_{k}, \boldsymbol{\Gamma}_{k}$, and $\boldsymbol{\Psi}_{k}$ can be seen as the sum of information from neighboring transmitters. See details for the linear transceiver case in [36]. However, such approach requires knowledge of all the interfering channel matrices to compute $\boldsymbol{\Upsilon}_{k}, \boldsymbol{\Phi}_{k}, \boldsymbol{\Gamma}_{k}$, and $\boldsymbol{\Psi}_{k}$.

An alternative procedure to obtain matrices $\boldsymbol{\Upsilon}_{k}, \boldsymbol{\Phi}_{k}, \boldsymbol{\Gamma}_{k}$, and $\boldsymbol{\Psi}_{k}$ is by exploiting uplink-downlink (UL-DL) propagation channel reciprocity, as is done in the proper signaling- 
based procedure in [6]. In that case, if we focus on DL interference coordination, matrices $\boldsymbol{\Upsilon}_{k}, \boldsymbol{\Gamma}_{k}, \boldsymbol{\Phi}_{k}$, and $\boldsymbol{\Psi}_{k}$, could be obtained from a UL pilot-based transmission provided that receivers use a specific pilot precoder for UL transmission that should be a function of the DL receive filters. By doing so, estimation of the interfering channels is not required and information exchange among transmitters is avoided. We omit the signal model here due to space limit. See more details for the linear transceiver case in [6][37], and for the widely linear transceiver case with minimum MSE criterion in [30].

\section{F. Multi-user MIMO extension}

The minWMSE problem $\left(\mathrm{P}_{1}\right)$ in (17) (as well as its decomposed problem $\left(\mathrm{P}_{1}^{k}\right)$ in (33)) can easily be extended to the case in which each transmitter serves multiple receivers simultaneously on the same time/frequency resource (multi-user MIMO systems). In this case, the power constraint associated to each transmitter couples the design of the (widely) linear transmit filters to be used to serve the associated receivers, but the solution can be derived by following the approach in Section III. See extension in [6] for the linear transceiver case.

\section{EVALUATIONS}

In this section we evaluate through Montecarlo simulations the performance of the proposed Algorithm 1 in Section III. The procedure is compared to the one in [4] that uses linear transceivers and proper Gaussian signaling (i.e. LP-LE).

A MIMO IC composed of $K$ transmitter-receiver pairs is considered. Channels are modeled through a Rayleigh distribution: $\mathbf{H}_{k k} \sim \mathcal{C N}(\mathbf{0}, \mathbf{I}), \forall k, \mathbf{H}_{k j} \sim \mathcal{C N}(\mathbf{0}, \eta \mathbf{I}), \forall j \neq k$, where factor $0<\eta \leq 1$ models the relative average interference strength. The noise is assumed to be white proper Gaussian, i.e. $\mathbf{n}_{k} \sim \mathcal{C N}\left(\mathbf{0}, \sigma^{2} \mathbf{I}\right), \forall k$. All transmitters are assumed to have the same available power $P_{k}=P, \forall k$, such that the signalto-noise ratio is defined by $\mathrm{SNR}=P / \sigma^{2}$. All transmitters (or receivers) are assumed to have the same number of antennas, which is denoted by $M$ (or $N$ ). Antenna configurations are depicted in figures as $M \times N$. 500 different channel realizations are averaged.

The following techniques are evaluated:

- LP-LE: interference coordination technique proposed in [4] with linear transceivers (LP-LE).

- LP-LE IW: iterative water-filling (IW) algorithm [38] with linear transceivers (LP-LE). It is equivalent to iteratively solve $\left(\mathrm{P}_{1}^{k}\right)$ in (33) at each $k$-th transmitter with $g_{k}=0$ (i.e. no coordination) and LP-LE.

- WLP-WLE: interference coordination technique proposed in this work with widely linear transceivers (WLPWLE). It is equivalent to use the composite real formulation and the technique proposed in [4].

- WLP-WLE IW: IW algorithm with widely linear transceivers (WLP-WLE). It is equivalent to iteratively solve $\left(\mathrm{P}_{1}^{k}\right)$ in (33) at each $k$-th transmitter with $g_{k}=0$ (i.e. no coordination) and WLP-WLE.

- HetTX $(l)$-WLE: interference coordination technique proposed in this work for a scenario of heterogeneous transmitters, whereby $\lfloor l\rfloor$ transmitters employ LP while

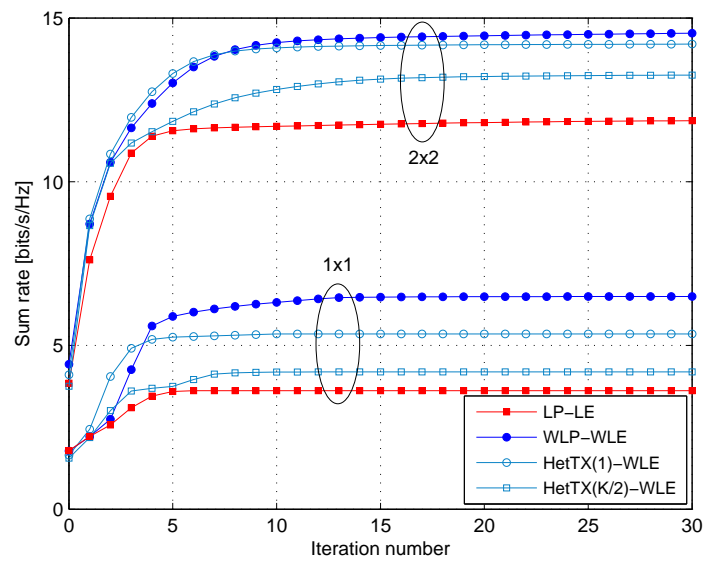

Fig. 2: Sum-rate (in bits $/ \mathrm{s} / \mathrm{Hz}$ ) vs. iteration number in a random channel realization of the $K$-user MIMO IC. $K=5, \mathrm{SNR}=10 \mathrm{~dB}, \eta=1$. Antenna configuration: $2 \times 2$ and $1 \times 1$.

the remaining $K-\lfloor l\rfloor$ transmitters can adopt WLP. $l=1$ and $l=K / 2$ are used for simulations.

The number of iterations of all algorithms is set to 50 , and the best result among 3 random transmit initializations is used. For the improper-based optimizations, a WLP initialization is required at least in some transmitters (i.e. random $\left\{\mathbf{T}_{1, k}, \mathbf{T}_{2, k}\right\}$ such that the power budget at each $k$-th transmitter is respected), otherwise the proposed procedure would lead to a proper Gaussian signaling solution (see discussion about initialization in Section III-B).

The performance indicator is the sum of achievable rates measured in bits/s/Hz. $\mu_{k}=1$ is used in (15), $\forall k$, except for Section IV-D.

In Section IV-A we show the convergence of Algorithm 1. The sum-rate performance is evaluated in Section IV-B for different antenna configurations when varying the number of transmitter-receiver pairs $(K)$ and the interference strength $(\eta)$. In Section IV-C, the proposed technique is compared with interference alignment schemes at different SNR regimes. Finally, in Section IV-D, a slightly modified version of Algorithm 1 is presented so as to guarantee fairness in the system, and the achieved performance is shown both in terms of sumrate and 5\%-tile rate.

\section{A. Convergence}

In this section we verify the convergence of the proposed Algorithm 1 for mixed transceiver design. The performance is compared to the one obtained for the linear transceiver case (i.e. LP-LE) in [4]. Fig. 2 shows the sum-rate versus the iteration number for a specific channel realization with antenna configurations $2 \times 2$ and $1 \times 1, K=5, \mathrm{SNR}=10 \mathrm{~dB}$, and $\eta=1$. Convergence in terms of sum-rate is observed. Further, monotonic convergence is obtained in this channel realization. Note that monotonic convergence is guaranteed in terms of the objective function of problem $\left(\mathrm{P}_{1}\right)$ in (17) but not necessarily in terms of sum-rate, although this is also often the case. The convergence speed is a bit slower for 'WLP-WLE' and 'HetTX-WLE' as compared to 'LP-LE', although it varies depending on the individual channel realizations. 


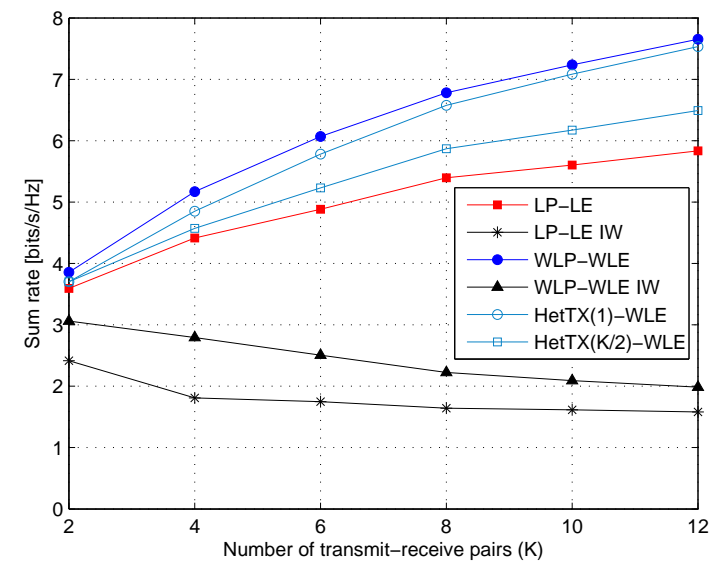

(a) $1 \times 1$

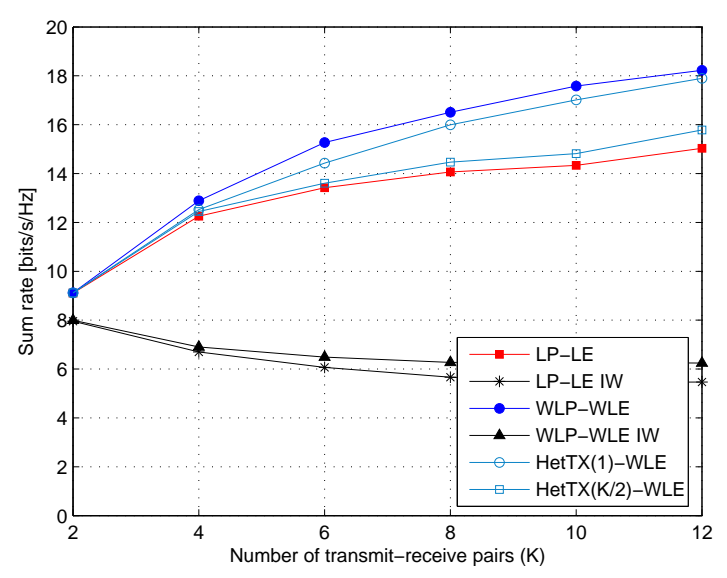

(b) $2 \times 2$

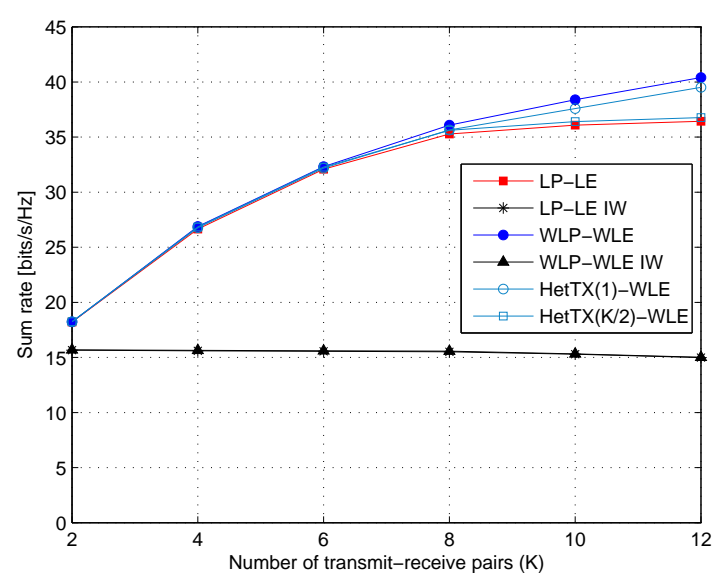

(c) $4 \times 4$

Fig. 3: Sum-rate (in bits/s/Hz) vs. $K$ for the $K$-user MIMO IC. SNR=10dB, $\eta=1$. Antenna configuration: (a) $1 \times 1$, (b) $2 \times 2$, (c) $4 \times 4$.

\section{B. Impact of antenna configuration and interference strength}

In this section we evaluate the performance of the proposed Algorithm 1 for different antenna configurations $(M \times N)$, different $K$ values, and different $\eta$ values. SNR $=10 \mathrm{~dB}$ is used.

Figure 3 displays the sum-rate versus $K$ for $\mathrm{SNR}=10 \mathrm{~dB}$, $\eta=1$, and different antenna configurations: Fig. 3.(a) for $1 \times 1$, Fig. 3.(b) for $2 \times 2$, and Fig. 3.(c) for $4 \times 4$. As it is expected, the sum-rate is increased as $K$ increases when

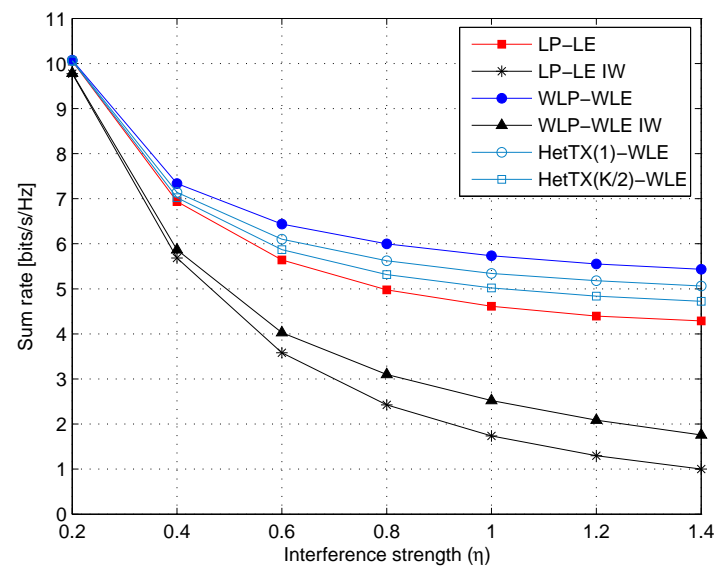

(a) $1 \times 1$

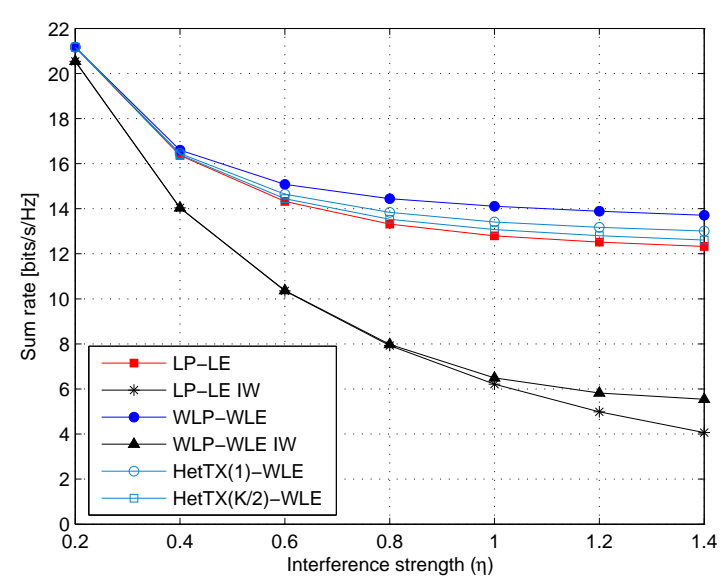

(b) $2 \times 2$

Fig. 4: Sum-rate (in bits/s/Hz) vs. $\eta$ (interference strength) for the $K$-user MIMO IC. $K=5, \mathrm{SNR}=10 \mathrm{~dB}$. Antenna configuration: (a) $1 \times 1$, (b) $2 \times 2$.

transmit coordination is implemented. However, for the cases in which transmit coordination is not used (i.e. IW solutions) the tendency is on the contrary because all transmitters use the maximum transmit power, such that increasing $K$ implies a significant increase of the interference in the network and, consequently, a degradation of the system performance.

Figure 4 depicts the sum-rate versus $\eta$ for $K=5$, $\mathrm{SNR}=10 \mathrm{~dB}$, and different antenna configurations: Fig. 4.(a) for $1 \times 1$, and Fig. 4.(b) for $2 \times 2$.

From Fig. 3 and Fig. 4 several conclusions can be extracted:

- Proper (LP-LE) vs. improper (WLP-WLE): In all cases it can be observed that 'WLP-WLE IW' outperforms 'LP-LE IW'. Also 'WLP-WLE' and 'HetTX-WLE' outperform 'LP-LE', as the proper-based optimization is a special case of the improper-based optimization (but not the other way round).

- IW vs. interference coordination: The sum-rate gain obtained with interference coordination techniques is significant for all antenna configurations, all numbers of interfering transmitters, and all interference levels.

- Number of transmitter-receiver pairs $(K)$ : The sum-rate increment provided by the use of improper Gaussian signaling is larger as $K$ increases due to the fact that 
the interference to be managed is stronger, see Fig. 3 . The $K$ value where the gains start to appear depends on the antenna configuration, as detailed in next bullet.

- Antenna configuration $(M \times N)$ : The sum-rate gain obtained with the use of improper Gaussian signaling is larger for the $1 \times 1$ and $2 \times 2$ cases rather than for the $4 \times 4$ case, because the use of improper Gaussian signaling provides flexibility by splitting one dimension into two halves. This is more useful when the number of transmit/receive antennas is low compared to the number of users. Otherwise, by adding antennas, extra dimensions are already added to the system. For that reason, the gains of interference coordination and improper Gaussian signaling are appreciable for $K \geq 4$ in the $2 \times 2$ case and for $K \geq 8$ in the $4 \times 4$ case, while in the $1 \times 1$ case they are substantial for $K \geq 2$ (i.e. even for a scenario with a single interferer), see Fig. 3.

- Interference strength $(\eta)$ : The sum-rate gain provided by improper Gaussian signaling is larger as the interference level increases (i.e. for larger $\eta$ ), see Fig. 4.

- Heterogeneous scenarios: The sum-rate gains of improper Gaussian signaling in heterogeneous scenarios are not proportional to the number of widely linear transmitters in the network. The largest gains are obtained when most of the transmitters are widely linear (i.e. lower $l$ in 'HetTX(l)-WLE').

\section{Performance versus SNR and comparison with IA}

In this section we evaluate the performance of the proposed Algorithm 1 at different SNR regimes. We compare the proposed technique with two algorithms based on interference alignment (IA) that are presented in [39]:

- IA: distributed iterative IA algorithm in [39].

- Max SINR: distributed Max-SINR algorithm in [39].

These two algorithms are optimal in terms of defrees of freedom (DoF) (i.e. slope of the sum-rate at the high SNR regime) for the MIMO IC with $K=3$ users and $2 \times 2$ antenna configuration, but not for other configurations.

Figure 5 shows the sum-rate versus SNR for $\eta=1,2 \times 2$ antenna configuration, and different $K$ values: Fig. 5.(a) for $K=3$, and Fig. 5.(b) for $K=5$.

For $K=3$ and $M=N=2$, 'IA' and 'Max SINR' algorithms attain the optimal DoF (i.e. $3 \mathrm{DoF}$ ), as shown in Fig. 5.(a). In contrast, the proposed scheme attains only $2 \mathrm{DoF}$ but it is able to provide significant sum-rate values at lowmedium SNR regimes. For $K=5$ and $M=N=2$, 'Max SINR' and 'IA' algorithms tend to become saturated in terms of sum-rate (thus 0 DoF), see Fig. 5.(b), while the proposed technique is able to get $2.2 \mathrm{DoF}$.

Many of the existing schemes for IA with improper Gaussian signaling are only valid for specific antenna configurations and number of users. On the contrary, the proposed scheme can be applied in all possible antenna/users configurations and still get some positive DoF.

For $K=5$, we can conclude that the gains of improper Gaussian signaling are appreciable for all SNR regimes. $K=3$ has been used for comparison with the IA algorithms,

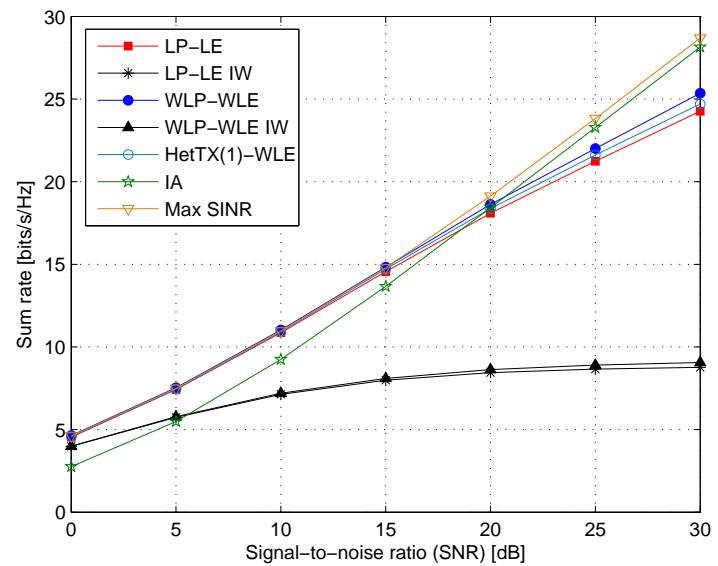

(a) $2 \times 2, K=3$

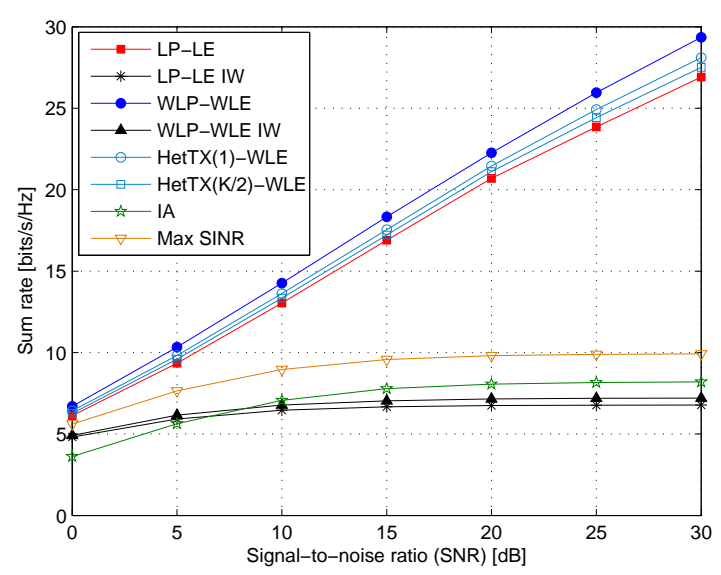

(b) $2 \times 2, K=5$

Fig. 5: Sum-rate (in bits/s/Hz) vs. SNR (in dB) for the $K$-user MIMO IC. $\eta=1$, $2 \times 2$. $K$ value: (a) $K=3$, (b) $K=5$.

but it was already shown in Fig. 3.(b) that in the $2 \times 2$ case we should use $K \geq 4$ to get appreciable gains with improper Gaussian signaling over 'LP-LE'.

\section{Performance with fairness utility}

In this section we evaluate the performance of a slightly modified version of the proposed Algorithm 1. In [4] it is shown that general utility functions can be accommodated in the WMSE problem formulation and only the design of the weighting matrices turns out to be affected. For example, in the linear transceiver design case, if a proportional fair utility function is adopted then the weighting matrices should be updated as: $\mathbf{W}_{1, k}=\frac{1}{R_{k} \ln (2)} \mathbf{E}_{k}^{-1}$ (see [4]). Similarly, in order to guarantee fairness in the system with mixed transceivers, we update the weighting matrices in Algorithm 1 as:

$$
\begin{aligned}
\mathbf{W}_{1, k} & =\frac{1}{R_{k} \ln (2)} \mathbf{F}_{k}^{-1} \\
\mathbf{W}_{2, k} & =-\frac{1}{R_{k} \ln (2)} \mathbf{E}_{k}^{-1} \tilde{\mathbf{E}}_{k} \mathbf{F}_{k}^{-*}
\end{aligned}
$$

being $R_{k}$ the achievable rate obtained in the previous iteration.

Figure 6 shows the performance in terms of sum-rate and $5 \%$-tile rate versus $K$ for $\mathrm{SNR}=10 \mathrm{~dB}, \eta=1$, and antenna configuration $2 \times 2$. It can be observed that 'WLP-WLE' and 'HetTX-WLE' techniques allow increasing the system fairness 


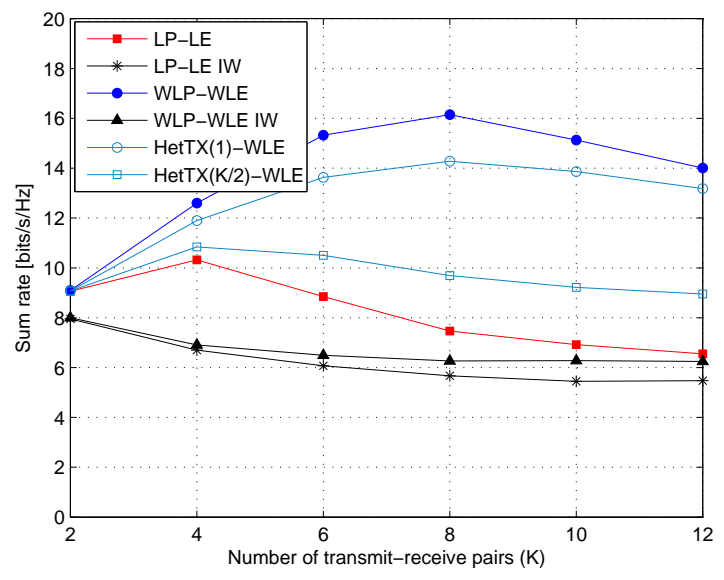

(a) sum-rate

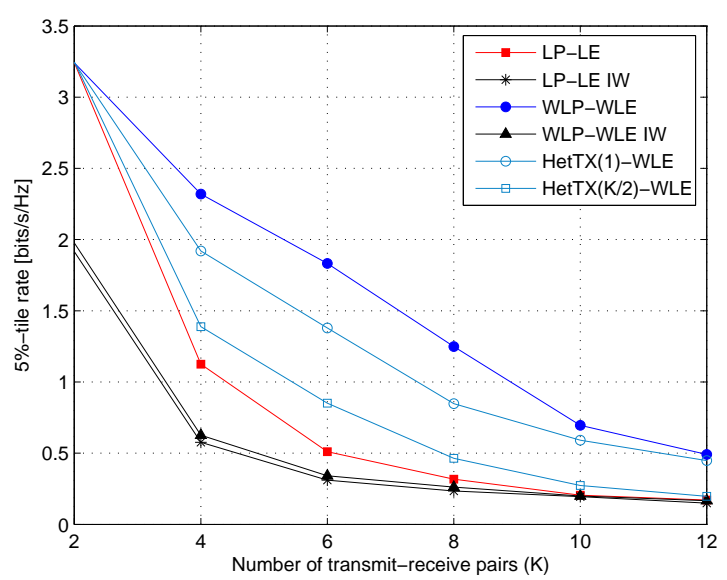

(b) $5 \%$-tile rate

Fig. 6: Performance vs. $K$ for the $K$-user MIMO IC. SNR=10dB, $\eta=1,2 \times 2$. Fairness utility. Performance metric: (a) sum-rate, (b) 5\%-tile rate.

(as shown in terms of the 5\%-tile rate in Fig. 6.(b)) while large gains in terms of sum-rate are still obtained (see Fig. 6.(a)).

\section{CONCLUSIONS}

In this article, transceiver design with improper Gaussian signaling for weighted sum-rate maximization in the $K$-user MIMO interference channel has been investigated. Maximization of the WSR is formulated using the complex-valued channel model, which allows covering a scenario where different types of transmitters (linear and widely linear) coexist. The initial maxWSR problem is solved through the minimization of an equivalent WMSE problem and closed-form expressions for mixed transceiver design are derived. In this regard, an iterative algorithm is presented which allows reaching a stationary point of the maxWSR problem. The proposed transceiver design (including transmit coordination and the use of improper Gaussian signaling) provides gains in terms of sum-rate as compared to conventional transmit coordination techniques with proper Gaussian signaling. The largest gains are observed in the following situations: 1) when the level of interference is high (either due to a small number of strong interferers or owing to multiple interfering nodes), or 2) when the number of transmit/receive antennas is low. If the interference level is not significant or new dimensions are provided by adding multiple antennas at transmitters and receivers, the proposed coordinated transceiver scheme leads to a proper Gaussian signaling solution.

\section{ACKNOWLEDGMENTS}

The authors would like to thank the anonymous reviewers for their constructive comments and valuable suggestions that have helped to improve the quality of the paper.

\section{APPENDIX A \\ PROOF OF THEOREM 1}

The equivalence of the minWMSE problem $\left(\mathrm{P}_{1}\right)$ in $(17)$ and the maxWSR problem $\left(\hat{\mathrm{P}}_{0}\right)$ in $(16)$ is shown in the following by deriving the optimal weighting matrices $\left\{\mathbf{W}_{1, k}\right\},\left\{\mathbf{W}_{2, k}\right\}$, for $\left(\mathrm{P}_{1}\right)$, plugging them into the objective function of $\left(\mathrm{P}_{1}\right)$, and then showing that the resulting optimization problem is exactly $\left(\hat{\mathrm{P}}_{0}\right)$.

The Lagrangian function $(\mathcal{L})$ [32] of the minWMSE problem $\left(P_{1}\right)$ in $(17)$ is given by:

$$
\begin{aligned}
\mathcal{L}= & \sum_{k \in \mathcal{K}}\left(\frac{1}{2} \operatorname{Tr}\left(\mathbf{W}_{1, k} \mathbf{E}_{k}+\mathbf{W}_{1, k}^{*} \mathbf{E}_{k}^{*}+\mathbf{W}_{2, k}^{*} \tilde{\mathbf{E}}_{k}+\mathbf{W}_{2, k} \tilde{\mathbf{E}}_{k}^{*}\right)\right. \\
& \left.\quad-\frac{\mu_{k}}{2} \log _{2}\left|\frac{\ln (2)}{\mu_{k}} \overline{\mathbf{W}}_{k}\right|\right) \\
& +\sum_{k \in \mathcal{K}} \lambda_{k}\left(\operatorname{Tr}\left(\mathbf{T}_{1, k} \mathbf{T}_{1, k}^{H}+\mathbf{T}_{2, k} \mathbf{T}_{2, k}^{H}\right)-P_{k}^{\max }\right)
\end{aligned}
$$

where $\lambda_{k}$ denotes the non-negative dual variable associated to the $k$-th transmit power constraint in (17). Recall that the determinant of $\frac{\ln (2)}{\mu_{k}} \overline{\mathbf{W}}_{k}$ in (38) (through the use of the structure in (18)) is given by:

$\left|\frac{\ln (2)}{\mu_{k}} \overline{\mathbf{W}}_{k}\right|=\left|\frac{\ln (2)}{\mu_{k}} \mathbf{W}_{1, k}\right|\left|\frac{\ln (2)}{\mu_{k}}\left(\mathbf{W}_{1, k}^{*}-\mathbf{W}_{2, k}^{*} \mathbf{W}_{1, k}^{-1} \mathbf{W}_{2, k}\right)\right|(39)$

The first-order optimality conditions for $\mathbf{W}_{1, k}$ and $\mathbf{W}_{2, k}$ lead to:

$$
\begin{aligned}
\frac{d \mathcal{L}}{d \mathbf{W}_{1, k}^{*}} & =\frac{1}{2} \mathbf{E}_{k}^{*}-\frac{\mu_{k}}{2 \ln (2)}\left(\mathbf{W}_{1, k}^{*}-\mathbf{W}_{2, k}^{*} \mathbf{W}_{1, k}^{-1} \mathbf{W}_{2, k}\right)^{-1}=\mathbf{0} \\
\frac{d \mathcal{L}}{d \mathbf{W}_{2, k}^{*}} & =\frac{1}{2} \tilde{\mathbf{E}}_{k} \\
& +\frac{\mu_{k}}{2 \ln (2)} \mathbf{W}_{1, k}^{-1} \mathbf{W}_{2, k}\left(\mathbf{W}_{1, k}^{*}-\mathbf{W}_{2, k}^{*} \mathbf{W}_{1, k}^{-1} \mathbf{W}_{2, k}\right)^{-1}=\mathbf{0}
\end{aligned}
$$

By identifying the expression in (40) inside (41) we have the following relation: $\tilde{\mathbf{E}}_{k}=-\mathbf{W}_{1, k}^{-1} \mathbf{W}_{2, k} \mathbf{E}_{k}^{*}$, such that: $\mathbf{W}_{2, k}=-\mathbf{W}_{1, k} \tilde{\mathbf{E}}_{k} \mathbf{E}_{k}^{-*}$. Then, by including such relation into (40) we obtain a closed-form expression for $\mathbf{W}_{1, k}$ as a function of $\left\{\mathbf{T}_{1, k}, \mathbf{T}_{2, k}\right\},\left\{\mathbf{R}_{1, k}, \mathbf{R}_{2, k}\right\}$. Similarly, by including $\mathbf{W}_{1, k}=$ $-\mathbf{W}_{2, k} \mathbf{E}_{k}^{*} \tilde{\mathbf{E}}_{k}^{-1}$ into (40) we obtain a closed-form expression for $\mathbf{W}_{2, k}$ as a function of $\left\{\mathbf{T}_{1, k}, \mathbf{T}_{2, k}\right\},\left\{\mathbf{R}_{1, k}, \mathbf{R}_{2, k}\right\}$. The optimal structures for $\mathbf{W}_{1, k}$ and $\mathbf{W}_{2, k}$ given all $\left\{\mathbf{T}_{1, k}, \mathbf{T}_{2, k}\right\}$, $\left\{\mathbf{R}_{1, k}, \mathbf{R}_{2, k}\right\}$ are:

$$
\begin{aligned}
\mathbf{W}_{1, k} & =\frac{\mu_{k}}{\ln (2)}\left(\mathbf{F}_{k}\right)^{-1} \\
\mathbf{W}_{2, k} & =-\frac{\mu_{k}}{\ln (2)} \mathbf{E}_{k}^{-1} \tilde{\mathbf{E}}_{k} \mathbf{F}_{k}^{-*}
\end{aligned}
$$

where $\mathbf{F}_{k}=\mathbf{E}_{k}-\tilde{\mathbf{E}}_{k} \mathbf{E}_{k}^{-*} \tilde{\mathbf{E}}_{k}^{*}$. 
Finally, by plugging the optimal $\mathbf{W}_{1, k}$ and $\mathbf{W}_{2, k}$ in (42)(43), $\forall k$, into the minWMSE problem $\left(\mathrm{P}_{1}\right)$ in (17), and using the determinant relation in (39) and the matrix inversion lemma, we have the following equivalent optimization problem:

$$
\begin{array}{ll}
\underset{\substack{\left\{\mathbf{T}_{1, k}, \mathbf{T}_{2, k}\right\} \\
\left\{\mathbf{R}_{1, k}, \mathbf{R}_{2, k}\right\}}}{\operatorname{minimize}} & \sum_{k \in \mathcal{K}} \frac{\mu_{k}}{2} \log _{2}\left|\mathbf{E}_{k} \mathbf{F}_{k}^{*}\right| \\
\text { subject to } & \left\{\begin{array}{l}
\operatorname{Tr}\left(\mathbf{T}_{1, k} \mathbf{T}_{1, k}^{H}+\mathbf{T}_{2, k} \mathbf{T}_{2, k}^{H}\right) \leq P_{k}^{\max } \quad \forall k \\
\mathbf{T}_{2, k}=\mathbf{0} \quad \forall k \in \mathcal{S}^{\mathrm{LP}}
\end{array}\right.
\end{array}
$$

which is equal to $\left(\hat{\mathrm{P}_{0}}\right)$ in (16) and completes the proof. This means that $\left(\hat{\mathrm{P}}_{0}\right)$ in (16) and $\left(\mathrm{P}_{1}\right)$ in (17) have the same global optimal solution $\left\{\mathbf{T}_{1, k}^{\star}, \mathbf{T}_{2, k}^{\star}, \mathbf{R}_{1, k}^{\star}, \mathbf{R}_{2, k}^{\star}\right\}$. Further, if $\left\{\mathbf{T}_{1, k}^{\star}, \mathbf{T}_{2, k}^{\star}, \mathbf{R}_{1, k}^{\star}, \mathbf{R}_{2, k}^{\star}, \mathbf{W}_{1, k}^{\star}, \mathbf{W}_{2, k}^{\star}\right\}$ is a stationary point of $\left(\mathrm{P}_{1}\right)$ then $\left\{\mathbf{T}_{1, k}^{\star}, \mathbf{T}_{2, k}^{\star}, \mathbf{R}_{1, k}^{\star}, \mathbf{R}_{2, k}^{\star}\right\}$ is a stationary point of $\left(\hat{\mathrm{P}}_{0}\right)$ as they satisfy the first-order optimality conditions of both problems. The converse also holds (i.e. if $\left\{\mathbf{T}_{1, k}^{\star}, \mathbf{T}_{2, k}^{\star}, \mathbf{R}_{1, k}^{\star}, \mathbf{R}_{2, k}^{\star}\right\}$ is a stationary point of $\left(\hat{\mathrm{P}}_{0}\right)$ then $\left\{\mathbf{T}_{1, k}^{\star}, \mathbf{T}_{2, k}^{\star}, \mathbf{R}_{1, k}^{\star}, \mathbf{R}_{2, k}^{\star}, \mathbf{W}_{1, k}^{\star}, \mathbf{W}_{2, k}^{\star}\right\}$ is a stationary point of $\left(\mathbf{P}_{1}\right)$ ), being $\left\{\mathbf{W}_{1, k}^{\star}, \mathbf{W}_{2, k}^{\star}\right\}$ the ones in (42)-(43), because for given $\left\{\mathbf{T}_{1, k}^{\star}, \mathbf{T}_{2, k}^{\star}, \mathbf{R}_{1, k}^{\star}, \mathbf{R}_{2, k}^{\star}\right\}$ then the optimal weighting matrices $\left\{\mathbf{W}_{1, k}^{\star}, \mathbf{W}_{2, k}^{\star}\right\}$ are those in (42)-(43).

To give more details about how we arrive at (44), let us show it for a specific receiver. First, by using the matrix inversion lemma, it can be shown that when including the structures of $\mathbf{W}_{1, k}$ and $\mathbf{W}_{2, k}$ in (42)-(43) into $\frac{1}{2} \operatorname{Tr}\left(\mathbf{W}_{1, k} \mathbf{E}_{k}+\mathbf{W}_{1, k}^{*} \mathbf{E}_{k}^{*}+\mathbf{W}_{2, k}^{*} \tilde{\mathbf{E}}_{k}+\mathbf{W}_{2, k} \tilde{\mathbf{E}}_{k}^{*}\right)$ in (17), then this term is a constant and can be taken out of the optimization. So let us focus on developing the term $-\frac{\mu_{k}}{2} \log _{2}\left|\frac{\ln (2)}{\mu_{k}} \overline{\mathbf{W}}_{k}\right|$ in (17) with the structures of $\mathbf{W}_{1, k}$ and $\mathbf{W}_{2, k}$ in (42)-(43). By using the determinant relation in (39) we have:

$$
\begin{aligned}
& -\frac{\mu_{k}}{2} \log _{2}\left|\frac{\ln (2)}{\mu_{k}} \overline{\mathbf{W}}_{k}\right| \\
& =-\frac{\mu_{k}}{2} \log _{2}\left|\mathbf{F}_{k}^{-1}\left(\mathbf{F}_{k}^{-*}-\mathbf{E}_{k}^{-*} \tilde{\mathbf{E}}_{k}^{*} \mathbf{E}_{k}^{-1} \tilde{\mathbf{E}}_{k} \mathbf{F}_{k}^{-*}\right)\right| \\
& =-\frac{\mu_{k}}{2} \log _{2}\left|\mathbf{F}_{k}^{-1} \mathbf{E}_{k}^{-*}\left(\mathbf{E}_{k}^{*}-\tilde{\mathbf{E}}_{k}^{*} \mathbf{E}_{k}^{-1} \tilde{\mathbf{E}}_{k}\right) \mathbf{F}_{k}^{-*}\right| \\
& =-\frac{\mu_{k}}{2} \log _{2}\left|\mathbf{F}_{k}^{-1} \mathbf{E}_{k}^{-*}\right|=-\frac{\mu_{k}}{2} \log _{2}\left|\mathbf{F}_{k}^{-*} \mathbf{E}_{k}^{-1}\right| \\
& =\frac{\mu_{k}}{2} \log _{2}\left|\mathbf{E}_{k} \mathbf{F}_{k}^{*}\right|
\end{aligned}
$$

such that the expression in (44) is obtained.

\section{APPENDIX B}

\section{PROOF OF PROPOSITION 2}

The optimal transmit filters, when keeping the remaining sets of variables fixed, are obtained by equaling the derivatives with respect to $\mathbf{T}_{1, k}^{*}$ and $\mathbf{T}_{2, k}^{*}$ of the Lagrangian function of the minWMSE problem $\left(\mathrm{P}_{1}\right)$ in (17) to zero. The Lagrangian function $(\mathcal{L})$ is shown in $(38)$. The derivatives result:

$$
\begin{aligned}
\frac{d \mathcal{L}}{d \mathbf{T}_{1, k}^{*}}= & -\mathbf{H}_{k k}^{H} \mathbf{R}_{1, k} \mathbf{W}_{1, k}-\mathbf{H}_{k k}^{H} \mathbf{R}_{2, k}^{*} \mathbf{W}_{2, k}^{*}+\left(\mathbf{B}_{k}+\mathbf{D}_{k}\right) \mathbf{T}_{2, k}^{*} \\
& +\left(\mathbf{A}_{k}+\mathbf{C}_{k}+\lambda_{k} \mathbf{I}\right) \mathbf{T}_{1, k}=\mathbf{0} \\
\frac{d \mathcal{L}}{d \mathbf{T}_{2, k}^{*}}= & -\mathbf{H}_{k k}^{H} \mathbf{R}_{2, k}^{*} \mathbf{W}_{1, k}^{*}-\mathbf{H}_{k k}^{H} \mathbf{R}_{1, k} \mathbf{W}_{2, k}+\left(\mathbf{B}_{k}+\mathbf{D}_{k}\right) \mathbf{T}_{1, k}^{*} \\
& +\left(\mathbf{A}_{k}+\mathbf{C}_{k}+\lambda_{k} \mathbf{I}\right) \mathbf{T}_{2, k}=\mathbf{0}
\end{aligned}
$$

where $\mathbf{A}_{k}, \mathbf{B}_{k}, \mathbf{C}_{k}$, and $\mathbf{D}_{k}$, are defined in (21), (23), (25), and (27), respectively.

This way, if we isolate $\mathbf{T}_{1, k}$ from (46) and substitute it to (47), we get an expression for $\mathbf{T}_{2, k}$ only as a function of $\left\{\mathbf{R}_{1, k}, \mathbf{R}_{2, k}\right\},\left\{\mathbf{W}_{1, k}, \mathbf{W}_{2, k}\right\}$. Similarly, if we isolate $\mathbf{T}_{2, k}$ from (47) and substitute it to (46), we obtain $\mathbf{T}_{1, k}$ as a function of $\left\{\mathbf{R}_{1, k}, \mathbf{R}_{2, k}\right\},\left\{\mathbf{W}_{1, k}, \mathbf{W}_{2, k}\right\}$. The derived expressions for $\mathbf{T}_{1, k}$ and $\mathbf{T}_{2, k}$ are those shown in (29)-(30).

In case the $k$-th transmitter is constrained to use LP, the solution for the transmit filter $\mathbf{T}_{1, k}$ is directly obtained from (46): $\mathbf{T}_{1, k}=\left(\mathbf{A}_{k}+\mathbf{C}_{k}+\lambda_{k} \mathbf{I}\right)^{-1}\left(\mathbf{H}_{k k}^{H} \mathbf{R}_{1, k} \mathbf{W}_{1, k}+\mathbf{H}_{k k}^{H} \mathbf{R}_{2, k}^{*} \mathbf{W}_{2, k}^{*}\right)$.

\section{REFERENCES}

[1] Z.-Q. Luo and S. Zhang, "Dynamic spectrum management: complexity and duality," IEEE J. Sel. Topics Signal Process., vol. 2, pp. 57-53, Feb. 2008.

[2] C. Shi, D. A. Schmidt, R. A. Berry, M. L. Honig, and W. Utschick, "Distributed interference pricing for the MIMO interference channel," IEEE Int. Conf. Commun., pp. 1-5, Jun. 2009.

[3] G. Scutari, D. P. Palomar, F. Facchinei, and J.-S. Pang, "Distributed dynamic pricing for MIMO interfering multiuser systems: a unified approach," IEEE Int. Conf. Network Games, Control and Optimization, Oct. 2011.

[4] Q. Shi, M. Razaviyayn, Z.-Q. Luo, and C. He, "An iteratively weighted MMSE approach to distributed sum-utility maximization for a MIMO interfering broadcast channel," IEEE Trans. Signal Process., vol. 59, pp. 4331-4340, Sep. 2011.

[5] S. Christensen, R. Agarwal, E. Carvalho, and J. M. Cioffi, "Weighted sum-rate maximization using weighted MMSE for MIMO-BC beamforming design," IEEE Trans. Wireless Commun., vol. 7, pp. 4792-4799, Dec. 2008.

[6] S. Lagen, A. Agustin, and J. Vidal, "Decentralized coordinated precoding for dense TDD small cell networks," IEEE Trans. Wireless Commun., vol. 14 , pp. 4546 - 4561, Aug. 2015.

[7] F. D. Neeser and J. L. Massey, "Proper complex random processes with applications to information theory," IEEE Trans. Inf. Theory, vol. 39, pp. 1293-1302, Jul 1993.

[8] I. Telatar, "Capacity of multi-antenna Gaussian channels," in $A T \& T$ Technical Memorandum, Jun. 1995.

[9] P. Viswanath and D. N. C. Tse, "Sum capacity of the vector Gaussian broadcast channel and uplink-downlink duality," IEEE Trans. Inf. Theory, vol. 49, pp. 1912-1921, Aug. 2003.

[10] P. Viswanath, N. Jindal, and A. Goldsmith, "Duality, achievable rates, and sum-rate capacity of Gaussian MIMO broadcast channels," IEEE Trans. Inf. Theory, vol. 49, pp. 2658-2668, Oct. 2003.

[11] C. Hellings, M. Joham, and W. Utschick, "QoS feasibility in MIMO broadcast channels with widely linear transceivers," IEEE Signal Process. Lett., vol. 20, pp. 1134-1137, Nov. 2013.

[12] C. Hellings and W. Utschick, "Iterative algorithms for transceiver design in MIMO broadcast channels with improper signaling," Proc. 10th Int. ITG Conf. Systems, Commun., Coding, Feb. 2015.

[13] C. Hellings and W. Utschick, "Performance gains due to improper signals in MIMO broadcast channels with widely linear transceivers,' IEEE Int. Conf. Acoustics, Speech and Signal Process., pp. 4379-4383, May 2013.

[14] Z. K. M. Ho and E. Jorswieck, "Improper Gaussian signaling on the two-uer SISO interference channel," IEEE Trans. Wireless Commun., vol. 11, pp. 3194-3203, Sep. 2012

[15] Y. Zeng, C. M. Yetis, E. Gunawan, Y. L. Guan, and R. Zhang, "Transmit optimization with improper Gaussian signaling for interference channels," IEEE Trans. Signal Process., vol. 61, pp. 2899-2913, Jun. 2013.

[16] Y. Zeng, R. Zhang, E. Gunawan, and Y. L. Guan, "Optimized transmission with improper Gaussian signaling in the K-user MISO interference channel," IEEE Trans. Wireless Commun., vol. 12, pp. 6303-6313, Dec. 2013.

[17] V. R. Cadambe, S. A. Jafar, and C. Wang, "Interference alignment with asymmetric complex signaling - Settling the Høst-Madsen-Nosratinia conjecture," IEEE Trans. Inf. Theory, vol. 56, pp. 4552-4565, Sep. 2010.

[18] S. A. Jafar, "Interference alignment: a new look at signal dimensions in a communication network," Foundations and Trends in Commun. and Inf. Theory, vol. 7, pp. 1-134, Jun. 2010. 
[19] C. Lameiro and I. Santamaria, "Degrees-of-freedom for the 4-user SISO interference channel with improper signaling," IEEE Int. Conf. Commun., pp. 3053-3057, Jun. 2013.

[20] P. J. Schreier and L. L. Scharf, Statistical signal processing of complexvalued data: the theory of improper and noncircular signals. Cambridge (UK), Cambridge University Press, 2010.

[21] G. Tauböck, "Complex-valued random vectors and channels: entropy, divergence, and capacity," IEEE Trans. Inf. Theory, vol. 58, pp. 27292744, May 2012.

[22] Y. C. Yoon and H. Leib, "Maximizing SNR in improper complex noise and applications to CDMA," IEEE Commun. Lett., vol. 1, pp. 5-8, Jan. 1997.

[23] W. Gerstacker, R. Schober, and A. Lampe, "Receivers with widely linear processing for frequency-selective channels," IEEE Trans. Commun., vol. 51, pp. 1512-1523, Sep. 2003.

[24] P. Bhat, S. Nagata, L. Campoy, I. Berberana, T. Derham, G. Liu, X. Shen, P. Zong, and J. Yang, "LTE-Advanced: an operator perspective," IEEE Commun. Mag., vol. 50, pp. 104-114, Feb. 2012.

[25] 3GPP TR 36.866, Study on network-assisted interference cancellation and suppression (NAIC) for LTE, Release 12, v12.0.0, Mar. 2014.

[26] C. Hellings and W. Utschick, "Block-skew-circulant matrices in complex-valued signal processing," IEEE Trans. Signal Process., vol. 63, pp. 2093-2107, Jan. 2015.

[27] E. Kurniawan and S. Sun, "Improper Gaussian signaling scheme for the Z-interference channel," IEEE Trans. Wireless Commun., vol. 14, pp. 3912 - 3923, Jul. 2015.

[28] S. Lagen, A. Agustin, and J. Vidal, "Improper Gaussian signaling for the Z-interference channel," IEEE Int. Conf. Acoustics, Speech, and Signal Process., pp. 1145-1149, May 2014.

[29] E. Dahlman, S. Parkvall, and J. Sköld, 4G: LTE/LTE-Advanced for mobile broadband. Elsevier Ltd, Academic Press, 2010.

[30] S. Lagen, A. Agustin, and J. Vidal, "Decentralized widely linear precoding design for the MIMO interference channel," IEEE Global Commun. Conf., Dec. 2014.

[31] D. P. Bertsekas, Nonlinear programming. 2nd ed. Belmong, MA: Athena Scientific, 1999.

[32] S. Boyd and L. Vandenbergue, Convex optimization. Cambridge, 2004.

[33] 3GPP TR 36.829, Enhanced performance requirement for LTE user equipment (UE), Release 11, v11.1.0, Dec. 2012.

[34] D. G. Luenberger and Y. Ye, Linear and nonlinear programming, Third edition. Springer, 2008.

[35] G. H. Golub and C. F. V. Loan, Matrix computations. The Johns Hopkins University Press, 1996.

[36] S. Lagen, A. Agustin, and J. Vidal, "Distributed inter-cluster interference management for CoMP-based cellular networks," IEEE Global Commun. Conf., pp. 4204-4209, Dec. 2013.

[37] S. Lagen, A. Agustin, and J. Vidal, "Decentralized beamforming with coordinated sounding for inter-cell interference management," European Wireless Conf., pp. 111-116, May 2014.

[38] G. Scutari, D. P. Palomar, and S. Barbarossa, "The MIMO iterative waterfilling algorithm," IEEE Trans. Signal Process., vol. 57, pp. 191753, May. 2009.

[39] K. Gomadam, V. R. Cadambe, and S. A. Jafar, "A distributed numerical approach to interference alignment and applications to wireless interference networks," IEEE Trans. Inf. Theory, vol. 57, pp. 3309-3322, Jun. 2011.

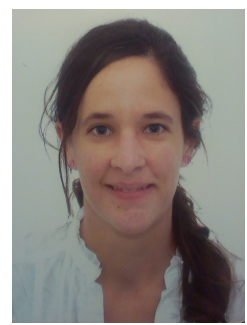

Sandra Lagen received the M.S. degree in Telecommunication Engineering from Universitat Politècnica de Catalunya (UPC), Barcelona, Spain, in 2011. In 2011 she joined the department of Signal Theory and Communications (TSC) at UPC as a research assistant, where she has participated in the EC funded projects FREEDOM and TROPIC. Since 2013 she is working towards her Ph.D. degree within the TSC department with an FPU grant from the Spanish Ministry of Education, Culture and Sport. Her research interests are focused on communication theory and signal processing for interference management in cellular networks.

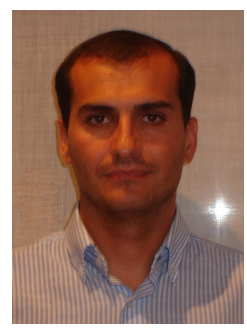

Adrian Agustin received the M.S. degrees in Telecommunication Engineering and Electronic Engineering and the Ph.D. degree from Universitat Politècnica de Catalunya (UPC), Barcelona, Spain, in 2000, 2002 and 2008, respectively. From 2000 to 2002, he was with Indra-Espacio, Barcelona, working on research and development of code synchronization techniques for DS-CDMA. In 2002 he joined the Signal Theory and Communications department at UPC as a research assistant. In 2008, he became a research associate. He has participated in the EC funded projects SATURN, ROMANTIK, FIREWORKS, ROCKET, FREEDOM, TROPIC and TUCAN3G. His research interests include wireless multi-user MIMO, interference management and interference alignment.

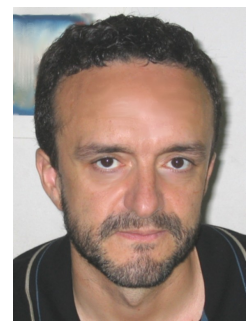

Josep Vidal received the Telecommunication Engineering and the $\mathrm{Ph}$. D. degrees from the Universitat Politcnica de Catalunya (UPC), Barcelona, where he is Professor at the Signal Theory and Communications department. His research interests are in statistical signal processing, information and communication theory, areas in which he has authored +150 journal and conference papers. Since 2002 has coordinated collaborative EC-funded projects ROMANTIK, FIREWORKS, ROCKET, FREEDOM, TROPIC and TUCAN3G, belonging to the FP5, FP6 and FP7 programmes, all in different areas of MIMO relay communications, self-organization, cooperative transmission and heterogeneous networks. He has held research appointments with EPF Lausanne, INP Toulouse and University of Hawaii, and has organized several international workshops. From 2011 through 2014 he served as associate editor of IEEE Transactions on Signal Processing. 